\title{
LHomme
}

L'HOMME Revue française d'anthropologie

212 | 2014

Varia

\section{Le singulier destin de Facing Mount Kenya. The Tribal Life of the Gikuyu (1938) de Jomo Kenyatta}

Une contribution à l'anthropologie des savoirs

The Singular Destiny of Facing Mount Kenya. The Tribal Life of the Gikuyu

(1938) by Jomo Kenyatta. A Contribution to the Anthropology of Knowledge

\section{Anne-Marie Peatrik}

\section{OpenEdition}

\section{Journals}

Édition électronique

URL : http://journals.openedition.org/lhomme/23737

DOI : $10.4000 /$ /homme.23737

ISSN : 1953-8103

Éditeur

Éditions de l'EHESS

\section{Édition imprimée}

Date de publication : 27 octobre 2014

Pagination : 71-108

ISSN : 0439-4216

Référence électronique

Anne-Marie Peatrik, "Le singulier destin de Facing Mount Kenya. The Tribal Life of the Gikuyu (1938) de Jomo Kenyatta », L'Homme [En ligne], 212 | 2014, mis en ligne le 24 octobre 2016, consulté le 02 mai 2019. URL : http://journals.openedition.org//homme/23737 ; DOI : 10.4000//homme.23737 


\title{
Le singulier destin de Facing Mount Kenya. The Tribal Life of the Gikuyu (1938) de Jomo Kenyatta
}

\author{
Une contribution à l'anthropologie des savoirs
}

\section{Anne-Marie Peatrik}

\section{$\mathrm{P}$}

OUR CEUX QUI ONT GRANDI durant les années de la décolonisation, le nom de Jomo Kenyatta, aux côtés d'autres associés bien souvent au conflit algérien, le plus marquant pour le public français, émaillait les bulletins radiophoniques et les titres de la grande presse. L'euphonie du nom du leader mau mau, le titre de son ouvrage ajoutaient à l'aura de la personne: Au pied du mont Kenya, comme au pied d'une montagne à gravir, d'un sommet à atteindre, rude mais belle promesse pour un peuple en route vers la radieuse aurore de l'indépendance.

Bien après, au cours d'une spécialisation en ethnologie, je redécouvris l'ouvrage dans sa version première dont le titre Facing Mount Kenya incite moins à l'escalade. Je le lus pour ce qu'il était, et est toujours à mes yeux, une monographie d'ethnologie sur les Kikuyu méridionaux que je croisais avec d'autres sources (Lambert 1956; Middleton \& Kershaw 1965; Kershaw 1972; Leakey 1977) pour trouver des données sur la question qui m'occupait alors: le démêlement des systèmes générationnels est-africains, plus particulièrement celui des Kikuyu (Peatrik 1994, 1995). Lors d'un échange informel à son sujet, John Lonsdale, spécialiste de

Une première version de ce travail intitulée «Le statut de l'anthropologie en Afrique orientale (Kenya)" a été présentée en mai 2011, au Séminaire général du Laboratoire d'ethnologie et de sociologie comparative "L'anthropologie en partage ? (CNRS - Université Paris Ouest Nanterre La Défense). Je remercie Christine Laurière, Daniel Fabre et André Mary pour leur invitation à exposer plus particulièrement sur le cas de Kenyatta le 24 avril 2012, dans leur séminaire à l'EHESS "Les ethnologues et le fait colonial " et pour les échanges stimulants qui s'ensuivirent. Enfin, j'exprime toute ma gratitude envers mes interlocuteurs au Kenya avec lesquels je me suis entretenue au sujet de Facing à l'automne 2011, principalement: Angela Kabiru, Nkirote M'MBogori, Suzana Mwongi, Chris Musambayi, Lucie Segero, Richard S. Odingo, Simiyu Wandibba, Henry Chakava. Les points de vue exposés ici n’engagent évidemment que ma seule responsabilité. 
l'histoire contemporaine kenyane, en présence de l'historien Godfrey Muriuki (1974) bien connu pour ses recherches sur les Kikuyu précoloniaux, fit l'éloge de mon article mais s’interrogea aussi sur la confiance que je paraissais accorder à l'ethnographie de Kenyatta. Un peu surprise, j'expliquai que certaines informations réputées farfelues, à l'usage d'une grande pertinence, attestaient la qualité de source ethnographique de Facing, mais j'accédai par là à une autre dimension de Facing, le jugement de défiance formulé à son endroit.

Facing, rappelons-le, est le fruit d'un master réalisé par Kenyatta de 1935 à 1937 à la London School Economic (LSE), sous la direction de Bronislaw Malinowski et publié en 1938. Salué comme la première enquête de ce genre et de cette qualité réalisée par un Africain, l'ouvrage est régulièrement évoqué dans l'histoire de l'anthropologie, à des fins illustratives diverses, dans le dossier, ô combien débattu, des liens entre domination coloniale et anthropologie ${ }^{1}$. Premier constat paradoxal : tout se serait passé comme si ce livre, pourtant écrit par un Kenyan devenu bien après premier président du Kenya indépendant (1964-1978), avait lui aussi contribué au rejet, dans les pays africains, de l'anthropologie en tant que science coloniale. De nos jours, au Kenya dans les années 2010, cette histoire paraît révolue, l'anthropologie sociale et culturelle jouissant d'un nouveau et plus enviable statut. L'ouvrage cependant continue de nourrir des sentiments ambigus, tantôt apprécié, souvent rejeté pour des raisons confuses. Certains commentaires, se faisant plus précis, pointent le caractère selfish de Facing qui, parlant du Kenya, ne parle que des Kikuyu. D'autres soulignent le rôle de ce texte dans l'affirmation du fondamentalisme des mungiki, bandes armées de jeunes Kikuyu impliquées, entre autres nombreuses milices, dans les violences postélectorales de 2007-2008.

Cette accumulation de jugements contradictoires révélatrice d'une destinée singulière indiquait un objet de recherche : reconstituer l'histoire de vie de Facing, à la manière des biographies of things (Kopytoff 1985) ou des "trajectoires d'objet", mais aussi des "lieux de mémoire» de Pierre Nora, et du Tour de France par deux enfants étudié par Jacques et Mona Ozouf $(1997 \text { [1984] })^{2}$. Et ce d'autant qu'à ma lecture d'ethnologue et aux

1. Les références à ce sujet sont légion. On peut évoquer par ordre chronologique : Gérard Leclerc (1972), Talal Asad (1973), Jean Copans (1975), pour la phase des débuts ; Jack Goody (1995), Adam Kuper (2000 [1996]), Benoît de L'Estoile (1997), en relais; Mwenda Ntarangwi, David Mills \& Mustapha Babiker (2006), David Mills (2008), Helen Tilley (2011) pour les références les plus récentes. Le temps aidant, les esprits semblent s'être apaisés.

2. Je remercie Christian Thibon, directeur de l'Institut français de recherche en Afrique, IfraNairobi, d'avoir ravivé cette source d'inspiration un peu enfouie dans ma mémoire, ainsi qu'Anouk Cohen dont les travaux sur le livre et l'édition au Maroc ont été une autre source d'inspiration,.../... 
informations recueillies par mes soins, je pouvais dorénavant ajouter les travaux d'historiens ${ }^{3}$ qui éclairent en particulier le contexte de la réalisation de l'ouvrage dans les années 1930 .

Pour avancer dans un tel projet, inédit à maints égards, il a fallu poser quelques garde-fous méthodologiques. La carrière d'un livre s'inscrit dans le champ d'intervention de trois catégories d'acteurs : la personne de l'écrivain et le moment de sa vie où il en a été l'auteur; les lecteurs, réels ou potentiels, destinataires de l'ouvrage; à la jointure de ces deux instances, l'éditeur responsable de la matérialité du livre et de sa diffusion. Par un effet de régression causale, cette quête biographique d'un ouvrage qui a traversé le XXe siècle et au-delà pouvait mener très loin dans l'épaisseur des événements et l'enchevêtrement des références. À ce premier risque de dispersion s'ajoutait celui, plus spécifique, consistant à rajouter une nouvelle couche interprétative sur un ouvrage déjà commenté à l'excès, alors que l'objectif ici est précisément de remettre au jour sa qualité de source ethnographique et d'inviter à le considérer comme tel. Pour sortir de cette intertextualité en abyme, l'accent sera mis plus particulièrement sur les avatars du livre en tant que produit éditorial, sur les échos rencontrés et les premières lectures, cela à trois moments clés de son existence : la monographie des années 1930, le livre étendard de la lutte anticoloniale, le livre de la discorde nationale.

\section{La scène londonienne et européenne dans les années 1930}

Qu'un sujet africain originaire de la colonie du Kenya, d'ascendance kikuyu, appelé Johnstone Kamau, arrivât à Londres en provenance de Mombasa au printemps 1929, cela n'allait pas de soi. Il fallait de bonnes raisons et quelques appuis pour entreprendre une telle croisière. Ce voyageur avait été envoyé par une association tribale, la Kikuyu Central Association (KCA), pour défendre directement auprès du Colonial Office, des parlementaires et de diverses commissions, des intérêts fonciers que le découpage des White Highlands au profit des colons européens, et au détriment des Kikuyu et d'autres aussi, avait gravement lésés.

[Suite de la note 2] et qui a soutenu sa thèse sous la direction de Jean-Charles Depaule, à Nanterre en 2011, et Anais Nin, doctorante, qui m’a aidée pour débusquer des textes inaccessibles. Pour une vue d'ensemble sur l'édition en Afrique, cf. Alain Ricard (1995).

3. La biographie de Kenyatta par Jeremy Murray-Brown (1972) fait toujours autorité. À l'exception de Ann Beck (1966), les travaux qui éclairent le contexte de la production de l'œuvre sont plus récents : Carlo Rossetti (1985); Woodford McClellan (1993); Bruce Berman (1996); Bruce Berman \& John Lonsdale (1991, 1998, 2007 ); Derek Peterson (2004 ); Bodil Frederiksen (2008). 
Âgé d'environ 35 ans, natif d'une localité proche de la station de chemin de fer devenue capitale de la colonie, Nairobi, Johnstone marié et père de deux enfants, appartenait à cette première génération de Kenyans africains convertis et scolarisés. Installé en ville, employé au service municipal des eaux, souvent sollicité pour sa capacité à parler, lire et écrire l'anglais, il avait fondé en 1928 avec d'autres compagnons, un journal mensuel écrit en kikuyu, Muigwithania ("Le Conciliateur»). Ce substantif désigne en kikuyu la personne capable de ramener la concorde, en l'occurrence dans les conflits de valeurs et d'intérêt générés par la domination coloniale.

À Londres cependant, les quelques appuis dont il disposait ne furent pas d'un grand secours, la cause des terres kikuyu n'intéressait guère de monde. Au terme d'un aller-retour de plusieurs mois au Kenya (septembre 1930-printemps 1931), pour rendre compte de ses déboires et trouver d'autres soutiens alors que la crise de la clitoridectomie battait son plein, Johnstone - après avoir débarqué à Gênes et être remonté par Paris où il visita l'Exposition coloniale de 1931 - prolongea son séjour, lequel allait durer encore quinze ans, mais cela, il ne le savait pas.

Sa quête de soutiens pour trouver des relais à ses revendications le conduisit à fréquenter tour à tour divers cercles progressistes de la capitale, des missionnaires, à commencer par la Church of Scotland Mission (Csm), Église où il avait été baptisé, et bientôt des politiques, divers courants travaillistes, des communistes, entrant également en contact avec d'autres Africains et des Antillais de l'Empire britannique installés à Londres. Des opportunités se présentèrent. À l'invitation de George Padmore, chantre à venir du panafricanisme qui n'avait pas encore démissionné du Komintern, il séjourna à deux reprises à Moscou, la seconde fois de l'automne 1932 au printemps 1933, dans une école de formation du Komintern. Séduit par l'anticolonialisme des communistes, il avait fini cependant par être catalogué de petit-bourgeois nationaliste: son attachement à une forme de " kikuyuitude " et à la propriété foncière était difficilement compatible avec la lutte des classes, mais son voyage au pays des Soviets et l'étiquette de communiste lui collèrent durablement à la peau. Enfin, une maîtrise croissante de l'anglais écrit, consolidée notamment lors de cours intensifs reçus dans le collège quaker de Woodbrooke College (septembre 1931-mars 1932), lui permit de commencer à publier dans des journaux britanniques sur la situation coloniale au Kenya, ce qui élargit là encore ses cercles de connaissances.

Mais ses revendications n'avançaient pas, la Morris Carter Land Commission déclara irrecevables les demandes de la KCA. Entre-temps, et pour gagner un peu d'argent, il était devenu informateur pour les 
linguistes qui étudiaient le kikuyu. À cette époque, un débat majeur sur l'écriture du kikuyu opposait les partisans d'une écriture à sept versus cinq phonèmes vocaliques (Peterson 2004). Ce travail avec les linguistes ouvrit la porte à d'autres possibles. Et en décembre 1934, Kenyatta finit par rencontrer Malinowski, titulaire de la chaire d'anthropologie sociale de la LSE. Le courant passa immédiatement, dit-on, peut-être parce que chacun pensait pouvoir compter sur l'autre. En suivant un enseignement dans cette toute nouvelle discipline qu'était l'anthropologie sociale, Johnstone voyait le moyen de consolider sa formation et de progresser dans le monde universitaire, façon de battre en brèche la soi-disant infériorité intellectuelle des Africains, manière aussi de se faire bien voir de l'intelligentsia africano-caraïbe de Londres; moyen enfin de poursuivre sa mission de défenseur des Kikuyu en présentant une description qui les rendrait intelligibles (Kenyatta 1938 : XVI-XVII).

Malinowski, rappelons-le, de retour d'Australie dans l'institution où il avait fait sa thèse avant la Grande Guerre, y enseignait l'anthropologie sociale depuis 1923, et s'était fait l'avocat d'une nouvelle approche fondée sur la recherche de terrain, l'observation participante et l'étude des populations réellement existantes. De plus en plus intéressé par l'Afrique, il s'était rapproché de l'International Institute for African Languages and Cultures (IIALC), créé en 1926, pour lequel, prônant une practical anthropology (Malinowski 1929), il avait œuvré auprès de la Rockefeller Foundation et obtenu des financements conséquents qui permirent de réaliser des enquêtes, au fondement de ce qui allait devenir une fameuse école d'anthropologie sociale.

Son projet de practical anthropology, mis au service du progrès dans les colonies, s'il n'a jamais été autre chose qu'un procédé rhétorique à l'appui de demandes de financement, avait de toute façon mal résisté au spectacle accablant de la situation coloniale, au cours d'un voyage qu'il avait pu enfin organiser (juillet-septembre 1934) en Afrique du Sud et de l'Est, au Kenya particulièrement. Lors d'un bref séjour chez les Kikuyu, il avait recueilli des informations sur les deux sources majeures de conflits générés par la colonisation: les revendications sur les terres et la coutume de la clitoridectomie. La possibilité d'approfondir ses analyses avec un Kikuyu lettré installé à Londres ne pouvait que l'intéresser. Il tenait son interlocuteur privilégié avec lequel - hypothèse personnelle -, ce Polonais originaire du défunt Empire austro-hongrois partageait une même interrogation quant à l'utilisation d'une langue écrite, l'anglais en l'occurrence, qui n'était pas sa langue maternelle. Quoi qu'il en soit, par un jeu d'équivalence et la possibilité de recruter à la LSE des étudiants ayant une "prior knowledge of natives situations ", suivant la clause introduite par 
Malinowski dans le règlement des études (Mills 2008: 38), celui-ci inscrivit Johnstone Kamau à son séminaire, dans un cursus de master étalé sur trois ans et obtint pour son étudiant une bourse de l'IIALC (ironie de l'histoire, le jury comme l'Institut était présidé par Lord Lugard en personne, qui rappelons-le, était le théoricien de l'indirect rule). C'est par l'entremise de Malinowski que Johnstone fit également un séjour d'une dizaine de jours à Paris en avril 1935, à l'invitation de la princesse Marie Bonaparte (Frederiksen 2008). Malinowski intéressé, on le sait, par la question de l'universalité du complexe d'Edipe, entretenait de nombreux échanges avec Marie Bonaparte, élève de Freud, connue pour ses analyses sur la libido féminine et l'origine de la frigidité. Faute de pouvoir aller elle-même en Afrique, la princesse invita Johnstone. Ils s'entretinrent de différents aspects de la clitoridectomie et il lui envoya ultérieurement un mémoire à ce sujet (Ibid. : 30-32).

$\mathrm{Au}$ titre de sa participation au séminaire de Malinowski, Kenyatta exposa plus particulièrement sur la propriété de la terre, l'initiation, l'éducation, la magie aussi, publia un article sur la religion (1937). Ces divers développements fournirent la matière du mémoire soutenu fin 1937, puis publié en 1938, après un travail éditorial normal ${ }^{4}$ (Berman 1996: 331).

Dans sa préface (in Kenyatta 1938 : VII-XIV), Malinowski salue l'auteur et l'accomplissement de son étudiant avec un tonitruant "Anthropology begins at home ", mais très vite son propos s'assombrit, marqué du sceau de la désillusion - «Progress is a terrible thing »-, de la montée de la guerre, de la lâcheté des démocraties face aux dictatures en Europe, en Éthiopie, en Chine... Malinowski et Kenyatta se sont rencontrés à des moments bien différents de leurs trajectoires personnelles. Quand le livre sort, Malinowski très démoralisé par les bruits de botte en Europe centrale (l'Anschluss date de mars 1938) est déjà parti aux États-Unis. Il y meurt en 1942.

\section{Le livre et sa première réception}

\section{Un produit éditorial}

Secker \& Warburg, maison d'édition tout juste fondée en 1936, dédiée à la littérature et aux textes politiques radicaux, envisageait de publier le premier ouvrage de recherche réalisé par un Afro-Américain, originaire de Trinidad, Cyril L. R. James, auteur de The Black Jacobins. Toussaint Louverture and the San Domingo Revolution. Sollicité à nouveau par les Afro-Antillais de Londres liés à l'Independant Labour Party, Frederic

4. Dinah Stock, chargée de cours dans une association de formation pour adultes (Workers Educational Association, WEA), membre du Labour et anti-impérialiste, l'avait aidé à corriger le tapuscrit de Facing (Murray-Brown 1972 : 199-200). 
Warburg accepta de publier le master de Kenyatta, d'autant qu'il connaissait bien Malinowski. Argonauts of the Western Pacific, entre autres ouvrages de l'anthropologue, avait été publié en 1922 par Routledge, maison où Warburg avait commencé sa carrière (Warburg 1959 : 89-90, 206, 251-254).

Le titre initialement proposé par l'auteur, Voice of the Gikuyu. Echoes from Mount Kenya (Murray-Brown 1972: 194), indiquait une sorte d'éloignement, un souci de réflexivité, dirait-on de nos jours. Le titre finalement retenu Facing Mount Kenya ramène l'auteur à son terroir natal et authentifie son témoignage. Nul ne sait qui a trouvé ce titre, assurément plus accrocheur, qui reproduit les derniers mots de la préface de Kenyatta (1938: XXI). Du sous-titre, The Tribal Life of the Gikuyu, il suffit de rappeler que "tribal» dénote moins la primitivité que son équivalent français et que de toute façon, sur le moment, il a paru aller de soi.

Du nom de l'auteur, en revanche, il y aurait beaucoup à dire. Déjà utilisé pour l'article de 1937, Jomo Kenyatta, euphonique et facile à retenir, est l'aboutissement de glissements et de choix successifs qui résument à eux seuls la vie de l'auteur, tour à tour ou simultanément appelé :

— «Kamau ", reçu à sa naissance, du nom du père de sa mère.

— Kamau "wa Ngengi », après son initiation, du nom du frère de son défunt père.

— «Johnstone" Kamau wa Ngengi après son baptême. Johnstone agrège les noms des deux apôtres choisis par le baptisé, John et Peter (traduit en Stone) pour conserver ces deux prénoms tout en en gardant qu'un seul, comme l'exigeaient les missionnaires.

- Johnstone Kamau "Kinyata», surnom évoquant les perles de la ceinture ouvragée à la façon maasaï qu'il aimait porter.

— «enyatta " résulte de la transcription approximative de « kinyata » ou "kinyatta", la ceinture ouvragée évoquée ci-dessus, qui permet un rapprochement avec « Kenya », le nom de la colonie.

— "Kenya », il faut le rappeler, résulte de la transcription approximative de « Kerenyaga » qui désigne le massif central kenyan : "Kere » signifie mont et "nyaga ", brillant ou lumineux, tacheté comme les plumes de l'autruche, en référence à la neige qui recouvre le sommet. "Kere-Nyaga " signifie "Mont Lumineux ", au sens de mont tacheté de blanc lumineux. Prononcé en kikamba, langue des Kamba qui sont les premiers locuteurs bantous en venant des régions côtières, et qui ont tendance à élider les consonnes en allongeant les voyelles, on obtient Ke[r]enya[g]a, Keenyaa, et en transcription simplifiée Kenya. Kenyatta a le mérite poétique de représenter/contenir par un procédé métonymique le "Kenya », nom d'une montagne qui désigne aussi toute la colonie. 
— «Jomo » diminutif de «Johnstone» recouvre un autre sens : « Jomo» se rapprocherait d'un substantif kikuyu signifiant " the pulling of a sword from its scabbard", le geste de tirer une épée de son fourreau, ce qui la fait bruire et briller (Murray-Brown 1972: 196). Rappelons que l'épée dans son fourreau tenu par une ceinture fait partie de l'attirail du guerrier de ces régions, ce qui nous renvoie à la photographie du frontispice commentée plus bas.

— "Jomo Kenyatta» : Jomo accolé à Kenyatta pourrait être traduit par "Épée qui luit lorsqu'on la tire de son fourreau du Kenya ", "Épée flamboyante du Kenya ». Seulement voilà, sur la photographie du frontispice, Jomo tient non pas une épée mais une lance: d'où la transformation de l'Épée lumineuse en "Javelot flamboyant " (Burning Spear)... du Kenya!

Ce jeu avec de multiples identités pourrait être analysé en termes de mise en scène de sa personne par un Kenyatta confronté à une altérité mouvante, ou encore d'une capacité d'adaptation mêlée d'opportunisme et d'un penchant pour les pseudonymes. Mais il reflète aussi, ou prolonge, la manière d'inventer des appellations de parenté et des dénominations propres aux institutions d'âge dont relèvent les Kikuyu. Un nouveau nom constitutif de la personne s'acquiert à l'avancée dans un échelon ou une classe, qui se cumule sans se substituer aux précédents. L'usage en adresse et en référence de chacun de ces noms, modulé suivant des codes plus ou moins contraignants, est l'objet d'un apprentissage.

La photographie du frontispice, face à la page de titre, fit là encore l'objet d'un choix soigneusement réfléchi. Mais autant le jeu sur son nom s'inscrit dans l'épistèmé de la personne kikuyu, autant le portrait résulte d'une tactique éditoriale. Réalisé par un photographe du nom de Brian Cobbold, le portrait campe l'auteur en guerrier ou en chef africain, Burning Spear, chef guerrier kikuyu appliqué à vérifier l'affûtage de sa lance et absorbé dans la préparation d'une attaque, message qui va étrangement résonner avec l'actualité, au moment de la crise mau mau.

Passons sur la coupe des cheveux, loin des usages des guerriers ou des anciens kikuyu. Arrêtons-nous sur le bouc: Jomo l'aurait laissé pousser en solidarité avec le negus Hailé Sélassié, barbu on s'en souvient, réfugié à Londres après l'invasion de son pays en 1936 par les troupes de l'Italie fasciste (Murray-Brown 1972 : 198). La cape de fourrure souple en peau de daman et de singe bleu est portée quant à elle à la manière des rois Ashanti ${ }^{5}$, et non point à la mode des Kikuyu qui se couvraient les épaules et le cou de leur pelisse, le plus souvent en peau de chèvre ou de mouton,

5. Je remercie Michèle Coquet pour m'avoir suggéré ce rapprochement avec les monarques ashanti. 


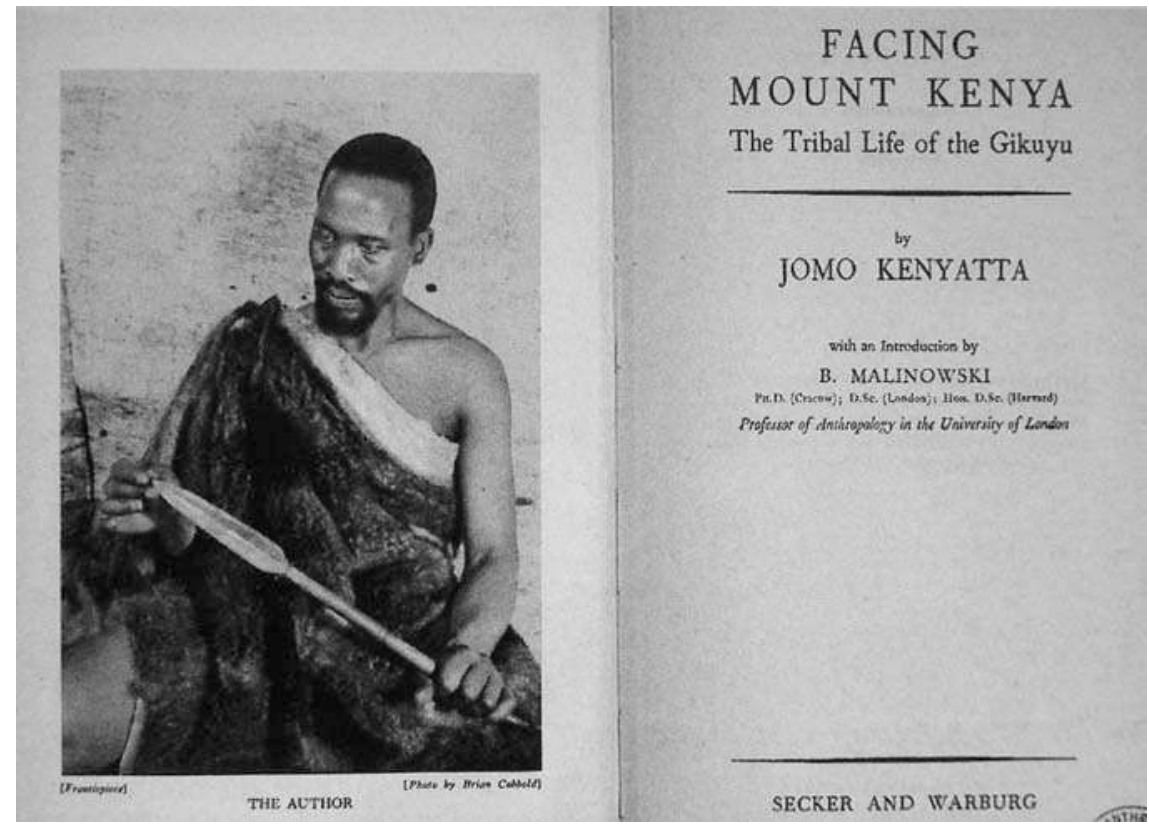

Fig. I. Portrait de l'auteur, frontispice in Facing Mount Kenya, 1938 (Cl. A.-M. Peatrik)

pour se tenir chaud. Le Ghana étant une colonie britannique, des portraits de rois ashantis perçus comme parangon du chef africain ont dû inspirer nos protagonistes. La lance à pointe courte parachève le cliché, mais accentue son caractère saugrenu, à contre-emploi de la bienséance kikuyu. Seuls arboraient leurs lances les initiés du plus bas de l'échelle de l'autorité, appelés les Kamatimo - les "Petits à la lance» - et il ne viendrait jamais à l'esprit d'un personnage d'importance (il n'y a pas de chefferie chez les Kikuyu) de se faire représenter de la sorte. Avec son bâton d'aîné certes, mais c'eût été moins évocateur pour les lecteurs de l'époque.

On a beaucoup glosé sur cette photographie qui a suscité des commentaires contraires. Un fait cependant est établi. Kenyatta était un grand amateur de photographies de sa personne, il s'habillait comme un dandy, aimait apparaître dans des costumes différents, comme il avait apprécié en 1935 de figurer dans un film d'Alexandre Korda, Sanders of the River, dans lequel tournait Paul Robeson, le premier acteur et chanteur noir célèbre (Ibid. : 185-187). Dans ce montage pour Facing, Kenyatta aurait bénéficié des conseils et de la complicité de Peter Mbiyu Koinange, fils du "chef " Koinange, de retour des États-Unis après avoir obtenu un «Bachelor of Art ». Il s'était rapproché de Kenyatta dans ces années-là et aurait prêté la cape de fourrure, trouvé le vieux sens de jomo, et confectionné ou déniché la lance de la photographie (Ibid.: 195-196). 
De toute façon, on comprend le choix éditorial. Outre que la photographie authentifie l'auteur, le portrait d'un Africain lettré qui n'a cessé d'être africain accentue l'effet de véracité intrinsèque au titre. Rendre l'ouvrage attractif, donner envie au lecteur qui le découvre en libraire de l'acheter. C'est bien l'objectif de tout éditeur auquel incombe la tâche de faire sa réclame et de vendre l'ouvrage ${ }^{6}$. D'autant que le contenu bien sérieux pouvait aussi paraître un peu ardu.

\section{Le contenu en contexte de Facing, une esquisse}

Une analyse détaillée du contenu de Facing n'aurait pas sa place ici. On donnera des éléments propres à remettre l'ouvrage dans le contexte des années 1930 et de l'anthropologie conduite durant cette période charnière, afin de permettre une lecture moins anachronique que celle à laquelle se livrent par exemple les historiens comme Bruce Berman (1996) ou John Lonsdale (Berman \& Lonsdale 1998, 2007) dans leur quête des origines et de la construction de l'ethnicité kikuyu. Leurs recherches - qui traitent d'un objet tout à fait légitime et qui ont mis au jour nombre d'informations sans lesquelles mon exploration n'aurait jamais pu exister -, à force de questionner le passé à la lumière du présent, laissent penser qu'il n'y aurait pas de place pour d'autres lectures. En outre, le procès en fonctionnalisme instruit à son encontre, là encore trop marqué du sceau des jugements rétrospectifs, produit les mêmes distorsions téléologiques et masque la richesse de son propos ${ }^{7}$.

Introduit par la préface de Malinowski puis par celle de Kenyatta (Kenyatta 1938: XV-XXI), le texte de 339 pages se compose de treize chapitres de taille inégale, suivis d'un glossaire et d'un index des thèmes et des noms de populations. En guise de bibliographie, quelques références indiquées en notes de bas page, en tout trois textes officiels et deux auteurs, Lord Lugard et Aristote. Le texte, écrit par un Kikuyu qui s'est présenté avec ses lettres de créances, est "la référence », autoréférentiel en somme, en écho à l'incipit de Malinowski "Anthropology begins at home». En illustration, huit planches de photographies hors texte et une carte schématique du Kikuyu Country, dont les contours paraissent aller de soi comme l'indiquent les premières lignes de la préface de Kenyatta et qui sont congruents avec la Kikuyu Land Unit de l'administration coloniale, alors qu'ils sont gros d'une controverse durable.

6. Nous n'avons retrouvé aucun exemplaire de la jaquette (dust-cover) de Facing. Jeremy MurrayBrown (1972: 376, n. 38) y fait allusion pour indiquer qu'elle annonçait la parution de Black Jacobins.

7. "Every page of Facing Mount Kenya makes plain that Kenyatta found in functionalist anthropology a convincing medium of representation» (Berman \& Lonsdale 2007 : 184). 


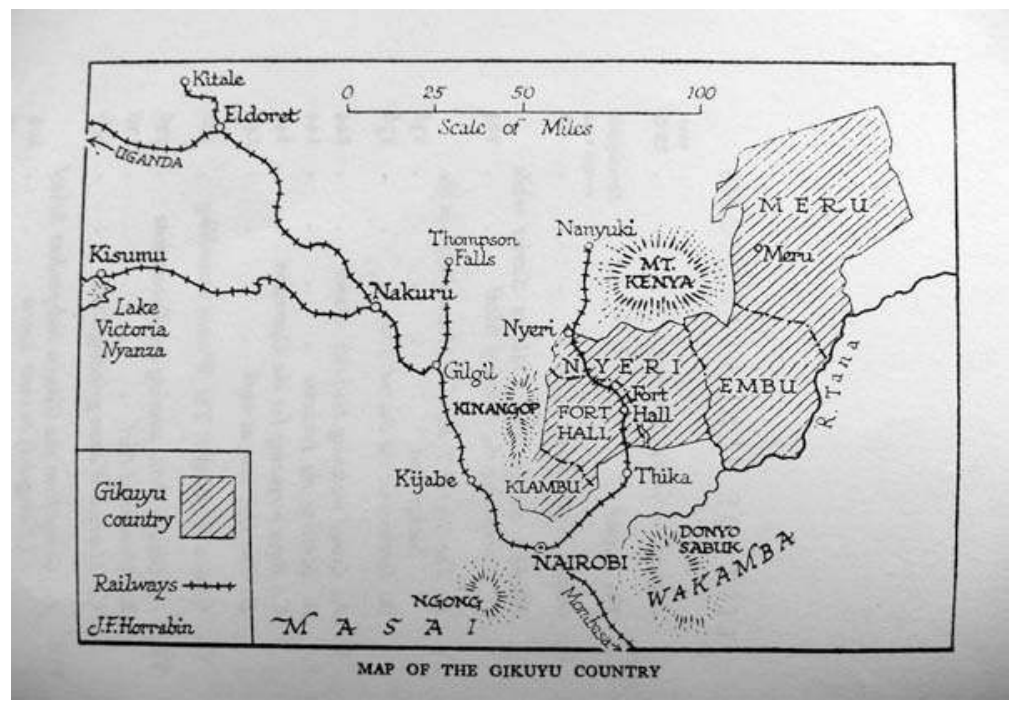

Fig. 2. Carte du pays kikuyu, en face de la page I, Facing Mount Kenya, I938 (Cl. A.-M. Peatrik)

Le plan enchaîne tour à tour la présentation des récits d'origine du peuple kikuyu et de l'organisation de la famille (Ibid.: chap. I : 20 p.), le droit foncier (Ibid. : chap. II : 32 p.), l'activité économique, agriculture, élevage, troc et artisanat y compris musical (Ibid. : chap. III et IV : 44 p.), les principes et les pratiques éducatives (Ibid. : chap. V: 30 p.), l'initiation des jeunes et les rituels féminins (Ibid. : chap. VI : 34 p.), la vie sexuelle prémaritale (Ibid.: chap. VII : 28 p.) et les institutions matrimoniales (Ibid. : chap. VIII : 22 p.), le système de gouvernement et les procédures judiciaires (Ibid. : chap. IX : 45 p.). Un dernier volet traite de la religion ancestrale (Ibid. : chap. X: 35 p.), des nouvelles religions (Ibid. : chap. XI : 10 p.), des pratiques magiques et médicales (Ibid. : chap. XII : 28 p.). Le dernier chapitre $(9$ p.), comme toute conclusion qui se respecte, rassemble ces différents aspects, mais dresse cependant un tableau plus cohérent que le corps du texte ne le suggère.

Ce qui frappe d'emblée à la lecture du texte est sa densité ${ }^{8}$. Facing en tant que monographie riche de données inédites fait regretter que des travaux de cette qualité n'aient pas été réalisés pour d'autres peuples du Kenya durant ces mêmes années, tant sont rares ou médiocres les ethnographies les concernant.

8. On mesure ainsi l'écart avec le bref écrit de Parmenas Mockerie (1934), l'autre délégué de la KCA lors du deuxième voyage à Londres au printemps 1931, dont l'opuscule de 65 pages a peut-être inspiré Kenyatta avec un résultat cependant qui n’a rien à voir. Cf. la recherche en cours intitulée: "Doubles et fantômes de Facing: les Kikuyu dans le miroir de l'ethnologie coloniale ". De la même façon, dans le chapitre VII de Facing, quelques rares éléments de la vie sexuelle prémaritale ont été empruntés à un article de Louis Leakey (1931) sans qu’il en soit fait mention. 
On pourrait rétorquer que cette monographie et sa visée d'exhaustivité ont bien vieilli. Avec son plan à tiroir qui envisage tous les aspects de la vie indigène "de la cave au grenier ", elle peut paraître désuète, soixante-dix ans après au cours desquels tant d'innovations conceptuelles et de découvertes empiriques se sont produites en anthropologie. Mais ce serait là encore porter un jugement anachronique.

Pour faciliter sa remise en contexte, la monographie gagne à être rapportée à l'article, déjà cité, de Malinowski, "Practical Anthropology ", en particulier les "new branches of anthropological inquiry" qu'il juge indispensables à toute monographie et couvrent la majorité de son article (1929 : 24-37). Tour à tour, il propose d'étudier en anthropologie politique, non pas seulement les rois sacrés et leurs rituels (la figure de Frazer n'est pas loin), mais aussi les institutions qui régissent ces monarchies et les pouvoirs; le droit coutumier, non en concevant d'emblée un individu complètement dominé par le groupe, mais en reliant sa condition à l'organisation sociale, la parenté, la famille, la communauté villageoise, la tribu ${ }^{9}$; puis la formation de la personnalité et l'éducation du primitif; l'étude concrète des langues en usage; l'anthropologie du droit foncier pour laquelle Malinovski préconise des cartes de parcellaire et recommande l'étude des conflits entre les intérêts des Blancs et ceux des Natives, ainsi qu'entre les intérêts des Natives eux-mêmes; l'organisation de l'économie, plus particulièrement la question du «travail ", celle du changement avec la diffusion des valeurs de l'Occident, de la westernisation, en bref l'anthropology of the changing Native.

De ce point de vue, il apparaît que Jomo s'est donc plié au programme de son maitre, et que la question foncière, qu'il avait évidemment à l'esprit, occupait aussi celui de Malinowski, et ce, bien avant leur rencontre! Et que le souci malinowskien de saisie synchronique, à l'encontre de la théorie évolutionniste de la survivance, n'excluait nullement l'étude du changement social auquel, outre ladite question foncière, conduisait le chapitre XI consacré à l'apparition des nouvelles religions comme les Watu wa Mngu.

L'exercice de remise en contexte pourrait se poursuivre dans une autre direction. Conjointement au relevé des catégories descriptives et analytiques d'inspiration malinowskienne, devenues de nos jours trop classiques, on pourrait aussi pointer des manques un peu étonnants dans un texte que caractériserait une visée d'exhaustivité. Ainsi, la chirurgie de la clitoridectomie est bien décrite, mais la circoncision que subissent les

9. À propos de la parenté, Malinowski énumère les catégories analytiques déjà bien établies: parenté classificatoire, organisation du clan, du groupe local, questions de filiation matrilinéaire et patrilinéaire. 
hommes ne l'est pas, et les photographies d'initiation (Kenyatta 1938: 144) prises à la demande de Jomo par son demi-frère (Ibid.: XVII) évoquent le rituel mais n'indiquent rien de précis. Était-il indécent, malséant de parler des garçons ou alors la circoncision de Kenyatta, pratiquée en milieu missionnaire (Murray-Brown 1972: 50-53), n'aurait-elle pas été tout à fait conforme à la coutume? Autre curiosité, Kenyatta indique bien son groupe d'âge initiatique ("kehiomwere», Kenyatta 1938 : XIX), mais ne dit rien de son appartenance générationnelle. Lacune supplémentaire, cette fois-ci à propos des rituels funéraires qui consistaient à abandonner les défunts aux hyènes et aux autres prédateurs. Serait-ce parce que Kenyatta, tôt orphelin de son père puis de sa mère, ne serait pas parvenu à objectiver ces moments douloureux et/ou qu'il paraissait difficile d'interpréter des usages bien contraires à la sensibilité des Occidentaux ? En ces cas jouent d'autres ressorts, à l'origine d'une difficulté à mobiliser et à restituer une ethnographie pour des raisons qui tiennent à la vie de l'ethnographe, à la fois informateur et enquêteur, mêlées à l'état très lacunaire des connaissances dans l'ethnologie des années 1930.

Après le registre des manques, le registre de la richesse et de la finesse de certaines descriptions, assurément "brutes de décoffrage " mais précisément à la disposition de quiconque saura en tirer parti : à propos des méthodes de production, de la possibilité qu’ont les épouses de disposer de leurs surplus agricoles, de la vitalité des échanges commerciaux, du rôle du bétail comme quasi-monnaie, du contrat permettant d'obtenir une terre, des modalités de la guerre. Autre lecture en creux : les développements sur la justice et les peines qui traitent de la transgression des interdits et des désobéissances, le rôle crucial des serments et les ordalies (Ibid. : 221-225, 301), à l'encontre de l'idée d'harmonie évoquée au fil des pages et reprise, il est vrai, dans une conclusion dont on peut se demander si Kenyatta en a vraiment été l'unique auteur.

Enfin et surtout, il faut souligner la description fine des institutions d'âge et de génération: ainsi de la continuité entre instance familiale et classes d'âge, non en raison d'un irrépressible penchant "fonctionnaliste " pour l'harmonie sociale, mais parce que la théorie lignago-centrée du recoupement des lignages par les classes d'âge n'avait pas encore été formulée ni opéré ses ravages analytiques dans la théorie du politique en Afrique de l'Est. À noter aussi des informations étonnantes mais avérées et cohérentes, comme la possibilité d'être encore guerrier à l'âge de 40 ans. Il faut avoir à l'esprit que Kenyatta (dans les chapitres I et IX, rappelonsle) tente de présenter des modalités d'organisation politique dont personne n'avait vraiment l'idée dans ces années (à l'exception de Marcel Mauss [1931]). Et s'il étaye bien la question de la passation des pouvoirs 
générationnels itwika, il tourne autour d'un concept kikuyu qui influence ses analyses mais qu'il ne parvient pas à expliciter : la souveraineté générationnelle bûrûri/tĥे, laquelle lui aurait permis d'aller encore plus loin dans ses propos anticolonialistes, à moins qu'elle ne l'eût obligé à reconsidérer l'extension territoriale du pays kikuyu telle qu'elle apparaît dans cette carte si problématique du "Gikuyu Country ".

Les African Political Systems édités en 1940 par Meyer Fortes et Evans Pritchard ne disent mot des organisations politiques générationnelles. Tribes Without Rulers en 1958, sous la direction de John Middleton et David Tait, ne nous renseigne pas davantage, même si Middleton, bien au fait de ces questions puisqu'il a coordonné les Ethnographic Survey du Kenya central de 1953, précise en introduction que les classes d'âge ne seront pas abordées. Les systèmes d'âge et de génération est-africains ne deviendront de vrais objets d'analyse (et autant de casse-têtes) qu'à partir des années 1960, voire 1970. Par ailleurs, le fait que les systèmes générationnels informent, au bas mot, l'idée d'une totalité intégrant l'ensemble des individus d'une société, conjointement avec l'idée et l'expérience que la génération montante exerce une pression et se révolte pour arriver au pouvoir, sont tout à fait dans le paradigme de ces systèmes (voir Peatrik 1994). Une bonne partie du procès instruit contre Kenyatta pour son fonctionnalisme repose sur cette méprise, ou plus exactement, sur cette méconnaissance. Idem pour la lecture des récits d'origine, charte sociale certes narrée à la manière de Jomo, mais dont tous les éléments existent dans les récits des autres peuples apparentés, y compris l'existence de « rois» tyranniques, en réalité des forgerons, ce qui nous ramène à des configurations répertoriées par ailleurs en Afrique de l'Est (Peatrik 1999: 335-379).

Enfin, il faut rappeler le double effet de critique anticoloniale produit par cette monographie. De par la simple description ordonnée de la société, à rebours de l'idée partagée par presque tous les colons selon laquelle ces populations primitives sont anarchiques et barbares ${ }^{10}$, cette monographie d'ethnologie est intrinsèquement anticoloniale ${ }^{11}$. A fortiori, tel serait le cas des chapitres ou des passages qui disent avec une grande

10. Un des lecteurs de l'ouvrage, John W. Arthur, missionnaire et médecin à Thogoto, établissement de la Church of Scotland Mission où le jeune Kenyatta arriva en 1909, et l'un des artisans les plus actifs contre la clitorectomie, s'exprimait ainsi dans une lettre, en décembre 1939: «What I remember is the Kikuyu filth, physical and moral, which produced smallpox, and terribly yaws sore bodies [pian], periods of famine, a people bound by fear of evil spirits, degraded, with polygamy and all that means in village life. All that is hidden J. K.'s book under a veneer of an ideal African home life. It is untrue " (cité in Murray-Brown 1972:362, n. 1).

11. Argument que Wendy James (1973) a judicieusement rappelé à une époque où les ethnologues étaient sommés de battre leur coulpe sur les liens entre colonialisme et anthropologie. 
économie de moyens le caractère absurde et inacceptable de la conquête impériale et de la domination des colons : la parabole du pauvre homme et de l'éléphant (Kenyatta 1938: 47-52), la contradiction inhérente à la domination coloniale (Ibid. : 197), ou les modalités de la guerre indigène comparées à celle des Occidentaux (Ibid. : 205-215).

\section{La première réception de Facing}

Selon Frederic Warburg (1959: 253), Facing a fait l'objet de comptes rendus élogieux. Dans la grande presse? Nous n'en avons pas la trace, mais dans des revues universitaires, trois au moins sont accessibles où se mêlent compliments et interrogations.

Le linguiste C. M. Doke, en 1938, dans Bantu Studies (Afrique du Sud) souligne le caractère exceptionnel du livre "one of the first Native Africans to write an anthropological monograph on his own people " (1938 : 245), célèbre la qualité de la recherche, sous la direction du professeur Malinowski, et celle des analyses et de l'expression anglaise, bien meilleures que dans l'ouvrage de John Soga (1930) sur les Xhosa traduit de cette dernière langue. En même temps, Doke formule deux critiques: l'une portant sur l'idée d'une supériorité de l'anthropologie "at home» avancée par Malinowski dans l'introduction, l'autre contre l'expression des «political feelings » et des critiques des États coloniaux et des missionnaires qui ponctuent le texte. Même bien fondés, ces propos n'en demeurent pas moins déplacés aux yeux de Doke, dans ce genre de recherche. Le linguiste spécialiste du kikuyu, Arthur R. Barlow dans Africa en 1939, mêle de la même façon éloges et critiques sur l'expression des sentiments anti-missionnaires et anti-administration ${ }^{12}$. Seul Cullen Young, en 1938, dans le Journal of the Royal African Society s'en tient aux éloges. En somme le décor est planté, les ambiguïtés potentielles - aux yeux des Occidentaux - sont décelées, mais tout cela aurait pu en rester là : nous ne sommes qu'en 1938.

Facing, imprimé à 2000 exemplaires, a été vendu à 517 copies. C'est un échec éditorial pour Warburg, et Kenyatta qui espérait des retombées financières des droits d'auteur. La période n'était pas favorable, pouvait-on vraiment l'oublier? Facing arrive dans les libraires en septembre 1938, au moment des accords de Munich et l'on peut comprendre que le lectorat potentiel avait l'esprit moins préoccupé par d'éventuelles études portant sur une tribu de l'Empire. Signe du destin peut-être, les 1500 exemplaires restant furent détruits lors d'un bombardement en 1941.

12. Arthur R. Barlow a un lien ancien avec Kenyatta. De même que John W. Arthur, il était missionnaire à Thogoto. Il a retrouvé Kenyatta à Londres en 1930 pour, entre autres, lui proposer de collaborer à son dictionnaire kikuyu-anglais. 
Kenyatta comme son livre sont donc pris dans la guerre. Il s'éloigne de Londres pour ne pas être enrégimenté, trouve un emploi dans une ferme comme travailleur agricole, épouse une Anglaise, Edna G. Clarke. Un garçon naîtra de cette union - avec une "Blanche »-impensable s'il était resté au Kenya. En 1942, sollicité par une association chrétienne qui souhaitait éditer des brochures sur les pays africains écrits par des Africains eux-mêmes (Murray-Brown 1972: 364, n. 7), il publie un opuscule My People of Kikuyu and the Life of Chief Wangombe, court (60 p.), mais étonnant car il annonce un changement d'écriture : l'auteur s'éloigne de la retenue propre au style universitaire, pratique une sorte de récit bien plus facile à lire que Facing, précise ou clarifie certaines analyses de la politique kikuyu, et entretient toujours la même confusion à propos de l'extension du pays kikuyu.

Au fil du conflit et de sa transformation en guerre mondiale qui repousse davantage son retour en Afrique, Kenyatta noue des liens de plus en plus étroits avec les Afro-Américains de Londres et l'International

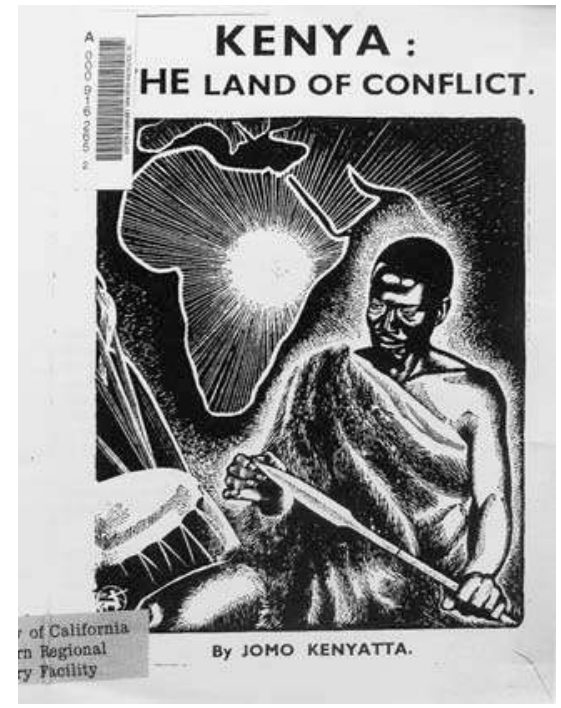
African Service Bureau (IASB). Née en 1935 de la crise éthiopienne, cette association regroupe des militants du panafricanisme auprès desquels la stature de Kenyatta grandit, en tant quauteur, en tant que politique et en tant qu'authentique Africain : c'est dans ce public que Facing trouve son premier lectorat ${ }^{13}$. Kenyatta, secondé par Padmore, dit-on (Ibid.: 221, 365), rédigea une autre brochure, de 26 pages, Kenya. The Land of Conflict (1945), éditée par l'IASB, qui n'a plus rien à voir avec Facing. C'est une brochure militante qui fait un historique un peu convenu mais virulent des méfaits de la conquête britannique. La couverture est toutefois décorée par une image-slogan extrapolée de la photographie de Burning Spear, et la parole de l'auteur est

Fig. 3. Première de couverture, Kenya...

(Cl. A.-M. Peatrik) légitimée de la sorte dans l'avant-propos: "Mr Kenyatta is the author of Facing Mount Kenya - an anthropological study on the tribal life of the Kikuyu - and is well qualified to speak for the Africans of Kenya ». Le signataire de cet éloge, George Padmore lui-même, est une des figures clés de l'anticolonialisme afro-antillais et anglophone (Boukari-Yabara 2014).

13. En écho, on pourrait évoquer le parcours parallèle de Léopold Senghor qui quitte le Sénégal en 1929, réussit l'agrégation de grammaire en 1935, assiste aux cours de Mauss et Rivet à l'Institut d'ethnologie. Ces années sont aussi celles de la rencontre avec Aimé Césaire arrivé de Fort de France, admis à Normale sup en 1935 et agrégé de lettres en 1939, et de la création de la revue L'Etudiant noir et de l'invention de la négritude. 
En octobre 1945 à Manchester, alors que la guerre est terminée, se tient le $\mathrm{V}^{\mathrm{e}}$ congrès panafricain où, témoignage de sa notoriété, Kenyatta occupe une place de choix. Il attend néanmoins encore un bon moment avant de s'embarquer, en septembre 1946, pour un voyage qui le ramène au Kenya, dans un pays qui s'est considérablement transformé durant les quinze années écoulées.

\section{Facing, le livre de l'anticolonialisme}

Facing comme étendard de la lutte anticoloniale est ce par quoi l'ouvrage est devenu célèbre, à tout le moins pour les générations qui ont grandi ou mûri à l'époque des décolonisations.

Dans le monde, comme dans les autres colonies, l'après-guerre 19391945 au Kenya fut lourde de nombreux orages. Le scénario de crise qui avait suivi la Grande Guerre se répéta avec plus d’intensité. La déception fut grande chez les Africains démobilisés qui retrouvèrent leur condition de dominés après avoir vécu et éprouvé une forme d'égalité dans les combats face à la mort. L'espoir d'une condition meilleure se dissipa d'autant plus rapidement que se produisit une nouvelle arrivée de colons blancs aggravant les tensions sur les patrimoines fonciers, dans le même temps où furent introduites des machines agricoles. Les "squatters" journaliers attachés aux domaines des White Settlers qui subsistaient grâce aux parcelles qu'on leur laissait cultiver, furent doublement confrontés à la perte de leur travail et à leur condition de sans-terre. Les barrières et la ségrégation raciales s'accentuèrent, laissant craindre un apartheid à la sudafricaine dont un arrêté stipulant que la langue de scolarisation des Africains serait désormais le kiswahili ne fut pas le moindre signe. Seule une petite élite aurait la possibilité de comprendre la langue des colons et de s'impliquer dans les échanges intellectuels internationaux.

De toute part sourdait l'opposition des Africains, légaliste avec la création en 1944 de la Kenyan African Union (KAU) dont Jomo Kenyatta fut élu président en 1947, diffuse puis violente à l'endroit de biens et de personnes, avec des actes de terreur bientôt attribués dans le courant de l'année 1948 à un mouvement dit Mau Mau. Le 20 octobre 1952, l'état d'urgence fut proclamé dans la colonie et l'armée envoyée d'Angleterre pour maintenir l'ordre au Kenya.

Avec cinq autres personnes, Kenyatta, arrêté et jugé au procès de Kapenguria - arrangé de toutes pièces et qui dura de fin décembre 1952 à début avril 1953 - fut condamné à neuf années de détention en tant qu'instigateur du Mau Mau - ce qu'il a toujours nié. Au cours des interrogatoires, Facing fut convoqué à la barre en ces termes : 
"- Anthony SOMerhough [procureur] : That was serious scientific study?

- JOMO KenYATTA : Yes.

— AS : Based on your personal knowledge and observations?

- JK: Yes.

- AS : Looking back on it, does it still represent your views on the Kikuyu Tribe?

- JK: The book cannot necessarily be my guide during my whole life.

- AS : Do you still regard it as a serious scientific work? Does it represent yours opinions?

- JK: It depends on what you mean.

— AS : I ask you, is it a serious scientific work ? Does it represent your opinions, and have you changed your opinions since then? Do you adhere to it?

- JK: The book is not my opinion as such, but it represents the habits and customs of the Kikuyu people.

- AS : Have you ever published a correction of the book or anything like that?

- JK: No, because I wanted to revise it when I came back, but having so many thing to do, I did not have the time.

— AS : And would you accept the description that the Crown applied : that you are an exceptionally widely travelled and exceptionally well educated African?

- JK: That is the Crown's opinion and you are entitled to your opinion» (Murray-Brown 1972: 268).

Singulière position assignée à ce texte, surprenant échange pour une soutenance d'un genre inédit entre un juré qui est un juge et son auteur un accusé. Les questions du juge et le style elliptique auquel se cantonne Kenyatta réferent à la controverse soulevée par Facing, directement associée à la crise mau mau et à des pratiques de serment et de meurtres dont les protagonistes furent au premier chef des Kikuyu.

Ailleurs, dans le monde, cette rébellion surprit, le Kenya était une colonie réputée calme. Le procès de Kapenguria auquel assistèrent des journalistes chevronnés ("best foreign correspondants of the English Press" [Ibid.: 260]), la renommée de son avocat, le sort réservé à Kenyatta, désormais un détenu politique ${ }^{14}$, transforma doublement sa position. D’opposant cantonné à un pays particulier, il se mua en héros de la lutte anticoloniale célèbre dans le monde entier. D'auteur lu dans un petit cénacle, il devint auteur à succès dont l'ouvrage fut mis dans les devantures des librairies : au titre de source d'informations sur les mystérieux Kikuyu et leurs étranges serments ; en tant que monographie d'ethnologie écrite par un sujet africain ; comme essai anticolonialiste prémonitoire dans sa dénonciation de l'appropriation des terres africaines par les Blancs. En bref, la rébellion mau mau et la crise coloniale furent une aubaine éditoriale pour Facing (Warburg 1959: 253-254). L'ouvrage,

14. Détenu dans des conditions très dures à Lokitaung dans la région des Turkana de 1952 à 1959, Kenyatta vit son sort s'améliorer à Lodwar (1960-1961), et enfin à Maralal jusqu’en août 1961. 
réimprimé dès 1952 à plusieurs reprises en édition reliée par Secker \& Warburg, le fut en édition brochée à partir de 1961, là encore objet de nombreuses réimpressions ${ }^{15}$. Facing fit sa percée aux États-Unis, et apparut dans les listes de lectures recommandées par les universités et les départements d'anthropologie ${ }^{16}$.

Autre preuve éloquente de sa nouvelle notoriété, Facing fut traduit en langue française. Pour répondre à l'intérêt suscité par la crise coloniale kenyane, un premier ouvrage sur Les Kikouyou et les Kamba du Kénia. Étude scientifique sur les Mau Mau de John Middleton fut publié par les éditions Payot, en 1954. Au pied du Mont Kenya parut en 1960. Le principal protagoniste de cette opération fut François Maspéro (2002: 171-197), éditeur concerné, s’il en est, par la crise algérienne et la décolonisation. Après avoir ouvert en 1955, au Quartier latin, sa librairie La Joie de lire, il avait fondé en 1959 les éditions Maspéro. La monographie de Kenyatta fut l'un des premiers titres qu'il publia.

Des deux traducteurs Pierre Balta et Gabriel Marcu, on peut dire qu'ils ont probablement travaillé dans l'urgence. Les termes choisis ne sont pas toujours très pensés ou pesés, le français paraît ramassé par rapport à l'anglais - ce qui est plutôt l'inverse d'habitude. On relève nombre de condensations, des coupures également, ainsi dans le chapitre XI consacré aux nouvelles religions qui écornent, il est vrai, l'image de tradition et d'ancienneté que l'ouvrage était censé véhiculer. Problème de droits peutêtre et de coût de revient, les photographies ont été supprimées; la carte (Keynyatta 1960: 22) a été conservée mais son titre - Carte des pays kikuyu - trahit une hésitation quant à l'extension et aux limites du territoire kikuyu. En revanche, le titre Au pied du mont Kenya sonne fort bien et, preuve que le nom de l'auteur et le titre parlaient suffisamment au lecteur potentiel, le sous-titre a été supprimé. Et s'il y avait encore quelque hésitation, la quatrième de couverture jouant à plein son rôle suscitait l'envie d'achat ${ }^{17}$. Relue un demi-siècle après, elle apparaît comme une excellente illustration, écrite par une main anonyme, de ce que l'on pouvait penser dans ces années :

15. Au point qu'il fut envisagé que les droits d'auteurs soient versés pour contribuer à l'éducation de son fils Magana Peter, né de son mariage avec Edna G. Clarke. Les démarches furent sans résultats, semble-t-il (Murray-Brown 1972:286;371, n. 7).

16. Depuis octobre 1965, à la suite d'un accord avec Secker \& Warburg, Facing est désormais édité dans la collection "Vintage Book» par Random House. Aux États-Unis, la renommée de Facing n'a cessé de s'amplifier au fil des décennies, sous l'effet de facteurs multiples. En guise d'illustration d'une recherche à venir, on peut citer un compte rendu de Facing paru en 2010, sous la plume masquée d'un sociologue bien connu de l'Université de Chicago, Barbara Celarent.

17. Cela dit sans ironie: rappelons que dans les années 1970, outre des démêlés sans fin avec les autorités de la République, la librairie a été la victime de vols « militants». 
"Ce livre se présente comme un traité d'ethnologie. Il décrit fidèlement la vie tribale des Gikuyu, habitants du Kenya. Sur ce point rien n'est omis dans cette relation passionnante, du système économique à la vie religieuse et spirituelle, en passant par les relations familiales et les rites d'initiation.

Mais la personnalité de l'auteur apporte à ce livre une autre valeur. Jomo Kenyatta ("Le javelot flamboyant du Kenya") retenu prisonnier depuis dix ans par les autorités anglaises, Africain du Kenya, Gikuyu né parmi les Gikuyu, est l'un des premiers leaders de l'indépendance africaine. C'est avant tout par amour de son peuple, pour le faire comprendre, en faire connaître la véritable civilisation, qu'il a patiemment construit son travail. Le traité scientifique se double donc d'une arme de combat. Grâce à Jomo Kenyatta, les Gikuyu - qui devaient donner naissance au mouvement Mau-Mau - apparaissent pour ce qu'ils sont : un peuple aux institutions harmonieuses et structurées, que l'invasion coloniale a brisées trop aveuglément. Et cette définition peut s'étendre facilement à l'ensemble des peuples africains.

Nous avons ici le tableau complet d'une culture africaine-type. Georges Balandier montre clairement dans sa préface, combien ce livre peut prendre une valeur exemplaire, au moment où, au Kenya comme au Congo voisin, les Africains affrontent violemment les problèmes posés par la "dé-civilisation" que plus de cent ans d'immixtion européenne leur ont infligée».

Est-il besoin de présenter l'auteur de la préface, George Balandier ? Le cadre restreint d'un article ne se prêterait guère à l'exercice ${ }^{18}$. Son texte remplace les introductions de Malinowski et de Kenyatta, évoquées de la sorte:

«La révolte Mau-Mau, et les charges portées à ce moment contre Kenyatta, ont plus fait pour forcer l'attention que l'introduction et la caution scientifique du regretté professeur Malinovski. La curiosité soudaine et la crainte, incitant à mieux connaitre l'ancien partenaire insurgé, ont vite consommé les éditions nouvelles » (Balandier in Kenyatta $1960: 8)$.

La nouvelle introduction, vigoureusement écrite, traduit l'urgence du moment et la fièvre de l'époque, la guerre d'Algérie est là en creux. Balandier donne des éléments de biographie du "Javelot flamboyant du Kenya ", et des indications sur la crise coloniale et la société kikuyu à partir d'un document qu'il cite souvent sans plus de précision : le Livre bleu, vraisemblablement le rapport commandité par le gouvernement britannique à Frank Corfield, The Origins and Growth of Mau Mau. An Historical Survey (1960)( "The Corfield Report»).

L'ouvrage se vend bien. Il inaugure, à un titre près, la collection «Textes à l'appui " appelée au succès que l'on sait. À cette époque, Jomo Kenyatta côtoie, entre autres, Paul Nizan (Les Chiens de garde, 1969 [1932]) et

18. On peut se référer à Georges Balandier, Georges Steinmetz \& Gisèle Sapiro (2011), dans l'attente de la publication des trois exposés présentés en 2012 au séminaire de l'Institut interdisciplinaire d'anthropologie du contemporain (IIAC, UMR 8177 CNRS EHESS), «Les ethnologues et le fait colonial » : André Mary "Le sociologue-ethnologue en situation : retour sur Afrique ambiguë»; Emmanuel Terray "Balandier, la colonisation, l'histoire "; Jean Jamin "Retour sur la révolte des Mau-Mau, Kenya, années $1950 »$. 
Vittorio Lanternari (Les Mouvements religieux de liberté et de salut des peuples opprimés, 1962). En 1967, quatre années après l'indépendance du Kenya acquise en 1963, Au pied du Mont Kenya fait l'objet d'une nouvelle édition et inaugure la "Petite collection Maspero", appelée elle aussi à un grand retentissement. En écho aux premières déconvenues postcoloniales, la préface de Georges Balandier est augmentée par son auteur de quelques paragraphes, dont l'extrait ci-dessous est indicatif d'un tournant dans l'étrange destinée de Facing:

"Jomo Kenyatta est au pouvoir, et le pouvoir l'a imprégné d'ambiguïté. L’image de celui que le Gouverneur du Kenya présentait encore, en 1960, comme "un guide vers les ténèbres et la mort" s'est transformée. Le héros national est devenu "le vieil homme" auquel les colons blancs font confiance, et The Economist lui consacrait un article, en mai 1965, sous le titre: "Notre homme au Kenya". Le nationaliste redouté s'efface derrière le gestionnaire "raisonnable", derrière le Président qui a ménagé les intérêts acquis, maintenu l'économie libérale et garanti la coexistence raciale. Les Hautes Terres ont été ouvertes aux paysans noirs, mais les exploitants européens sont restés sur leurs domaines et la compétition n'opère pas sur la base de l'égalité des chances. Le pouvoir blanc n'est pas aboli, et quelques-uns des anciens compagnons de Kenyatta lui reprochent avec vigueur sa modération" (Kenyatta $1967: 17$ ).

En somme, à la faveur de cette rencontre inopinée mais frontale de Facing avec l'Histoire, le texte est lu, parcouru, suscitant irritation ou enthousiasme. Quelles interprétations ont pu déposer sur le texte les attentes d'un lectorat élargi et fort hétérogène ${ }^{19}$ ?

La plus virulente mais la moins durable fut la lecture d'une bonne partie des colons kenyans. Incapables d'envisager la moindre autonomie pour les natives, décillés ou sidérés par la rébellion et la terreur mau mau - un parallèle avec la décolonisation de l'Algérie s'impose ici -, les colons identifient en Kenyatta et Facing, agents à leurs yeux de la propagande communiste, la source de tous leurs maux. L'histoire leur a donné tort. La rébellion contre les White Settlers devint aussi une guerre civile (Kershaw 1997). L'indépendance acquise en 1963, Kenyatta reprit son bâton de conciliateur et invita à tourner la page. Favorable à la scolarisation, à la caféiculture et à l'unité nationale, il fit paraître sous sa direction, en 1968,

19. Étant donné le statut international de la langue française dans ces années-là, la traduction chez Maspero contribua à diffuser encore plus largement Facing. En 1973, Le Club africain du livre, en accord avec François Maspero, édite Au pied du mont Kenya. L'ouvrage fut traduit en italien en 1976, sous le titre La Montagna dello Splendore. Per un'antropologia dell'Africa nera, et réédité en 1990. Apparemment, rien de tel en allemand, en portugais, espagnol, chinois ou russe. Il n'est pas impossible que l'ouvrage ait été traduit par les Japonais qui, comme les Italiens, ont depuis longtemps une active politique de traduction. Dans ce tour du monde (inachevé) des traductions de Facing, je remercie pour leur concours Erdmute Alber, Michel Cahen, Laurence Caillet, René Collignon, Giordano Marmone, Marie-Dominique Mouton. Cf. aussi infra, la note 28, pour la traduction de Facing en kiswahili. 
un recueil composé d'un volet biographique suivi de ses discours entre 1963 et 1967, intitule Suffering Without Bitterness (The Founding of the Kenya Nation), révélateur d'une modération parfois amèrement reprochée.

D'une tout autre portée fut le hiatus entre la «barbarie» des pratiques mau mau et l'idée d'organisation et de concorde sociale émanant du texte de Jomo : la question du lien entre la coutume et la rébellion des Kikuyu, et partant, la véracité de Facing et la validité de l'approche anthropologique furent tout à la fois questionnées, alimentant des controverses durables à l'origine de plusieurs courants interprétatifs. La controverse "anthropologie et colonialisme" atteint là un paroxysme. En quelques mots d'une recherche en cours (voir supra, note 8), rappelons le désaccord et le conflit de légitimité entre Jomo Kenyatta et Louis $\mathrm{S}$. Leakey à propos de la connaissance et de la représentation des Kikuyu (Berman \& Lonsdale 1991), et l'engagement de ce dernier dans des campagnes anti Mau Mau où il préconisait des contre-serments inspirés, pensait-il, de la coutume et destinés aux militants jugés amendables ${ }^{20}$ (Leakey 1952, 1954). À l'indépendance, Kenyatta ne lui tint pas rigueur de sa collaboration active avec les autorités coloniales, le paléo-anthropologue put continuer de développer, et ses recherches et le musée national de Nairobi. Se tenant à l'écart, des ethnologues anglophones s'employèrent à améliorer les connaissances sur les populations du Kenya central s'attachant, enfin pourrait-on dire, à démêler l'amalgame des peuples de la province centrale doublement réunis par l'administration et par Kenyatta, sous la bannière de la Kikuyu Land Unit ${ }^{21}$.

Dernier courant intellectuel né de cette confrontation de Facing avec le Mau Mau, l'interprétation de la rébellion et de la décolonisation en termes de "messianisme révolutionnaire ${ }^{22}$ ou de prophétisme. Cette interprétation se nourrit d'éléments de Facing, telle la prophétie indépendantiste narrée sous forme de conte dans le chapitre II, ou encore le sort réservé à son auteur, condamné dans un procès inique, voué aux

20. Cf. : Adriaan Prins (1953), Harold Lambert (1956), John Middleton (1953), Greta Kershaw (1972). Il faut aussi évoquer les recherches africanistes francophones sur les liens entre coutume et rébellion mau mau : Robert Buijtenhuijs dont la thèse est publiée en 1971, Le Mouvement "Mau Mau». Une révolte paysanne et anti-coloniale en Afrique noire met en avant la question de l'effet du «itwika " générationnel ; Jean Jamin (1977). Enfin, pour la critique ou le dépassement du fonctionnalisme malinowskien par les nouvelles écoles d'anthropologie à partir des années 1950, on pourra consulter les bons manuels d'histoire de l'ethnologie.

21. Louis S. Leakey (1903-1972) né en pays kikuyu, et parlant parfaitement la langue, avait réalisé une ethnographie des Kikuyu dont il s'était inspiré pour les deux ouvrages de 1953 et 1954, et qui parut en 1977, après sa mort donc.

22. Suivant l'expression retenue pour traduire le titre de l'ouvrage de Wilhelm Mühlman (1968 [1961] : 82-118). La relecture, à cinquante ans de distance, du chapitre consacré au Mau Mau laisse pantois tant il transpire la caricature. 
enfers de la détention avant d'être promu leader providentiel au-dessus de la bataille. La personne de Kenyatta, politique têtu et pragmatique, dandy lettré et bon vivant, cadre mal avec le rôle du prophète que ce récit lui assigne, mais pour le coup cette version rencontrera nombre d'échos, en raison notamment de la superposition de deux modalités de l'attente, l'attente prophétique des Églises indépendantes et l'attente, très mondaine, de la passation générationnelle (itwika).

\section{Facing au Kenya : de la concorde à la discorde nationales}

Pour éclairer le dernier avatar, à la date de cette recherche, dans la carrière décidément pleine de rebondissements de Facing, deux points de vue complémentaires seront croisés : celui de la diffusion de l'ouvrage dans le pays d'origine de son auteur alors que ce dernier en est devenu le premier président ; la lecture qu'en auraient faite les Mungiki, un mouvement de jeunesse kikuyu impliqué dans les émeutes post-électorales et les massacres de 2007-2008.

La diffusion de Facing au Kenya relève d'abord de l'état de l'édition au Kenya. Dès les années qui ont suivi l'indépendance, l'industrie du livre a été florissante au Kenya, en lien direct avec le développement continu de la scolarisation. L'enjeu de l'éducation des Africains qui avait tant mobilisé les esprits au moment des luttes anticoloniales mobilise maintenant l'énergie des citoyens kenyans. Grâce aux revenus du café, le pays s'équipe en écoles primaires bientôt complétées par un réseau d'écoles secondaires en internat. L'édition scolaire devient et demeure le moteur de l'édition tout court.

Lidée d'éditer Facing au Kenya revient à Henry Chakava ${ }^{23}$, un acteur clé de l'édition au Kenya qui, dans les années suivant l'indépendance était responsable de la branche Kenya du groupe Heinemann of Publishers, lequel avait absorbé Secker \& Warburg en 1951 (Warburg 1973: 136146). Chakava a monté deux opérations sur Facing du vivant de Kenyatta.

\section{La version scolaire de Facing (197I)}

Parmi toutes sortes d'ouvrages scolaires existaient, pour les écoliers de la fin du primaire et du secondaire, des listes de livres recommandés par le ministère de l'Éducation pour les examens nationaux (School Reading List). Or, toujours selon Chakava, ces ouvrages achetés à l'avance par le 
ministère étaient ensuite livrés directement dans les écoles. L'éditeur était donc assuré de la vente d'une certaine quantité d'ouvrages. Dans ce contexte a germé l'idée de réaliser une version scolaire de Facing, plus courte et se voulant plus accessible. La première de couverture de ce volume réduit à 169 pages qui paraît en 1971 est présentée sur la figure 4 .

Sur fond rouge est esquissé le mont Kenya, surmonté à gauche par le portrait en noir et blanc d'un Kenyatta âgé, à la trempe un peu prophétique faisant face au titre de son ouvrage. L'indication "School Edition " est bien

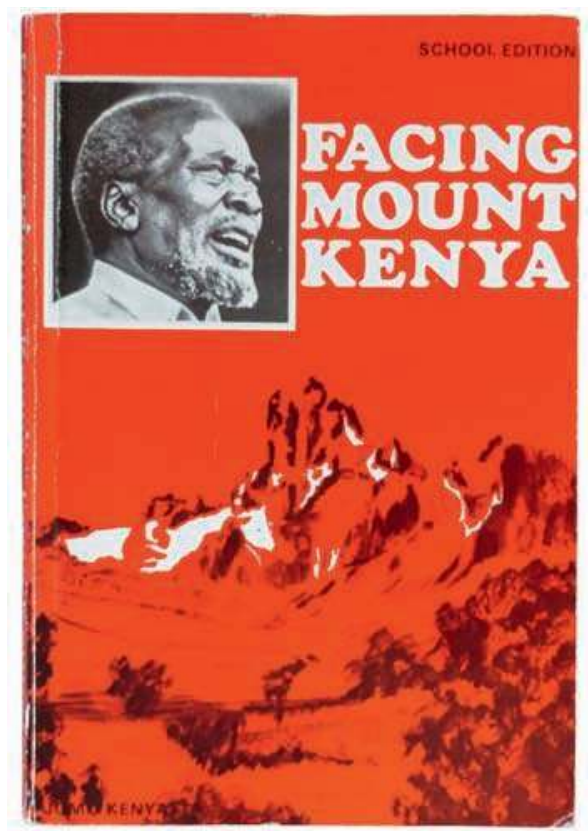

Fig. 4. Première de couverture, Facing Mount Kenya, I97I ("School Edition”) (Cl. A.-M. Peatrik) lisible et le nom familier de l'auteur est noyé en bas à gauche dans l'illustration. Sur la page de titre interne au volume apparaissent le sous-titre non modifié The Tribal Life of the Gikuyu, ainsi que le nom de l'auteur de cette version scolaire réduite, J. Kariuki.

Un court avant-propos de son Excellence Mzee Jomo Kenyatta remplace les deux préfaces de 1938. Kenyatta n'y prononce pas le nom d' "anthropologie» mais rappelle la raison qui l'a poussé à faire cet ouvrage :

«[...] to produce a book on some aspects of African tradition and culture, which would make impact on those who had no real knowledge of how Africans lived and thought and organised their own societies. It had often seemed frustrating to represent, or negotiate on behalf of, people only barely visualided under headings of character or personality " (Kenyatta $1971: 1$ ).

Dans cette note que l'on peut rapprocher de la formule de l'édition de 1938, l'auteur témoigne à nouveau, en des termes plus synthétiques, de la même intention, se faire mieux comprendre de ses interlocuteurs.

Dans cette version abrégée, le nombre de chapitres est passé de 13 à 11, glossaire et index ont été supprimés (cf. le tableau en annexe). Chaque chapitre se clôt par des exercices destinés aux élèves. Les illustrations ont été renouvelées, avec des photographies de paysages contemporains, par exemple. La carte initiale est conservée, à quelques détails près, ce qui surprend un peu. Pour donner une idée des coupes ou des condensations opérées et leurs effets possibles sur le lecteur, deux exemples sont présentés.

Ainsi le chapitre I, "Tribal Origin and Kinship System " (Ibid.: 1-11) a été réduit d'un gros quart (169 lignes sur 583). L'économie d'ensemble cependant a été respectée : l'exposé des récits d'origine est suivi de la présentation des principaux caractères de la morphologie sociale, groupes 
de filiation et mariage, puis institutions d'âge et de génération. Le contenu des passages réduits ou supprimés tout comme certains ajouts laissent transparaître certaines intentions.

La description vivante que donne Kenyatta de la manière dont les hommes ont circonvenu les femmes est réduite et vidée de ses allusions sexuelles, en même temps que disparaît la teneur récréative de ce récit (Ibid. : 6-7). La suppression des commentaires comparatifs sur les attitudes entre parents (Ibid.: 8) ou encore des données fines sur les termes de parenté (Ibid.: 11, 12) simplifie certes le contenu mais là encore vide le chapitre de sa substance, d'autant que s'il est un domaine où des élèves pourraient très concrètement saisir des différences culturelles, c'est bien celui de l'appellation des parents. Cette suppression est aussi révélatrice de la grande ignorance où se tenaient (et se tiennent) les personnes " éduquées" au Kenya qui ne soupçonnaient même plus que le vocabulaire de parenté puisse avoir eu de l'importance. L'effet des définitions ajoutées en bas de page accentue le caractère simplificateur jusqu'à la caricature du texte. Le matriarcat (Ibid.: 3) est ainsi défini : " a type of society in which the mother is the head of the family ", ce qui a pour effet de transformer une spéculation de l'évolutionnisme du XIXe siècle (le matriarcat) en savoir certifié. Autre exemple (Ibid.: 5), la définition de "patrilineal : possessions pass to the males in a family" réduit le problème de la filiation à la transmission des biens alors que - doit-on le rappeler? - la patrilinéarité touche à maints autres aspects. Erronées ou tronquées, ces définitions figent la pensée en une doxa.

Le traitement de ce premier chapitre est exemplaire de la méthode employée à des fins "pédagogiques». Certes, toute édition scolaire est par nature simplificatrice, mais, dans ce cas, le travail a vraiment eu des effets pernicieux. Là où la lecture de l'ethnographie pouvait induire une réflexion salutaire sur les savoirs ethnologiques, la version école rabote les interrogations et la portée du texte. Cela est d'autant plus frappant que le souci pédagogique transparaît bien dans les questions et les exercices proposés à l'élève à la fin du chapitre, comme s'il y avait eu un remords ou une tentative ultérieure de corriger les effets appauvrissant du texte ${ }^{24}$.

24. "Things to do: 1/At the beginning of this chapter you read the legend of Gikuyu and Moombi. Compare this legend with the stories of the beginning of your own people or of any other people you know about. You could also compare and contrast this legend with the story of the Creation in the Old Testament. (Class discussion). 2/Your parents have probably helped a great deal in your upbringing. Describe what your parents have done for you. What do you think you owe your parents in return? (Essay). 3/Write a brief character sketch of your mother or father (or a close relative). Describe physical appearance and personality and show what part the person you have chosen plays in your family's life. Further reading: Some of you will have already read.../... 
Le sort réservé au volet, ô combien débattu, de l'initiation et du mariage, soit trois chapitres dans la version initiale, fournit la seconde illustration. Dans la version Kenyatta, le chapitre VI «Initiation of Boys and Girls " (Kenyatta 1938 : 130-154) est évidemment un chapitre crucial étant donné le conflit sur la clitoridectomie déjà évoqué. Dans l'édition scolaire, le chapitre VI, conservé mais très raccourci, fait une dizaine de pages contre 24 pages dans l'édition de 1938 : des pans entiers ont été gommés, des titres reformulés. Supprimées la présentation des origines de la crise des années 1930, l'évocation du mouvement des écoles indépendantes (Gikuyu independant schools, Kareng'a schools), en somme les moyens intellectuels de contextualiser et d'historiciser. Disparues les descriptions des gestes pré- et post-opératoires, rituels ou pragmatiques sur lesquels Kenyatta insistait puisque c'est précisément à propos des désastres chirurgicaux subséquents qu'avait commencé la polémique. Les deux soustitres How the Girl is Operated on (Ibid. : 143-147) et Healing of the Wound (Ibid.: 147-153) sont remplacés par Initiation (Kenyatta 1971: 81-86) suivi de questions qui procèdent par allusions. On perce bien l'intention de l'auteur de l'édition scolaire. Il lui était difficile à la fois de s'adresser à un public scolaire sur ces questions, et d'escamoter ce volet, pour une bonne partie à l'origine de la recherche de Kenyatta. Kariuki s'y prend de la façon la plus euphémisée possible et évoque dans les questions des coutumes sur le déclin, avec cependant le non-dit persistant au sujet de la circoncision des garçons. Le chapitre VII, "Sex Life Among Young People» est carrément supprimé. De ce fait, le chapitre sur l'initiation est directement suivi par celui du mariage "Marriage System ", induisant une déformation majeure dans la présentation des faits. À ce point, on se demande à quels objectifs répondait l'idée d'une version scolaire de ce texte. Était-ce une idée sensée ? Kenyatta, qui a rédigé la nouvelle préface, l'aurait-il même lue ? ${ }^{25}$

Quel a été l'impact de cette édition scolaire? Après la parution initiale en 1971, les réimpressions se sont succédé à un rythme rapproché - en 1972, 1973, 1975, 1977 -, à chaque fois avec un tirage compris entre 5000 et 10000 exemplaires. Entre 1978 et 1990, en raison d'un conflit entre l'auteur de la version scolaire et l'éditeur, les réimpressions ont été

[Suite de la note 24] "Things Fall Apart" by the Nigerian writer Chinua Achebe (Heinemann Educational Books: African Writers Series). You will find it very helpful to compare the customs and traditions of the Gikuyu with those of the Ibo. Try to read this book while reading "Facing Mount Kenya" " (Kenyatta $1971: 12$ ).

25. Les années 1968-1969 sont un tournant dans la présidence de Kenyatta. Victime d'une crise cardiaque, Kenyatta est obligé de ralentir sensiblement ses activités. En juillet 1969, Tom Mboya, un des politiques les plus prometteurs de sa génération, critique de certaines orientations prises par le régime, est assassiné. Ses funérailles sont l'occasion de manifestations virulentes, complètement imprévues, à l'encontre de Kenyatta venu assister aux cérémonies dans l'ouest du Kenya, en région luo. 
suspendues ${ }^{26}$ puis elles reprennent en 1991 et en 2011, avec des tirages revus à la baisse (en moyenne 3000 exemplaires). Cette décrue dans la diffusion, mais non sa disparition, est mise en relation par l'éditeur avec la réforme du système éducatif de 1985 (dite "eight/four/four») qui a entraîné la suppression des listes nationales de livres de lecture. Les choix s'opèrent désormais au niveau local par chaque école et les livres sont obtenus via le réseau des librairies, nouvelle organisation du marché où l'éditeur peut moins anticiper.

L'édition intégrale (1978)

Dans le même temps où se finalisait l'édition scolaire, Henry Chakava, peu satisfait du travail car jugeant que trop d'aspects intéressants avaient été escamotés, envisagea de publier au Kenya la version intégrale de Facing. Warburg donna son accord, céda à Heinemann Kenya les 700 exemplaires qui lui restaient, lequel les écoula localement tout en préparant une nouvelle édition. Sur ces entrefaites, survint en 1978 le décès de Kenyatta, événement qui accéléra le processus, et l'ouvrage parut cette même année.

La première de couverture, sur fond bleu en négatif du mont Kenya, présente dans un cadre ovale le portrait de Jomo à Maralal en 1960, à la fin de sa détention. La distance parcourue et le temps écoulé depuis le chef-guerrier de 1938 sont clairement signifiés. Le texte lui-même n'est en rien modifié, il n'y a que de menues retouches. Le terme "tribal» devenu désobligeant dans ces années, le soustitre The Tribal Life of the Gikuyu se transforme en The Traditional Life of the Gikuyu. L'illustration, mise au goût du jour, inclut des photographies de Jomo détenu puis de Jomo Président et, surtout, manière de clore le débat sur l'extension du «Kikuyu Country ", la carte qui laisse entendre que le pays kikuyu va jusqu'à inclure

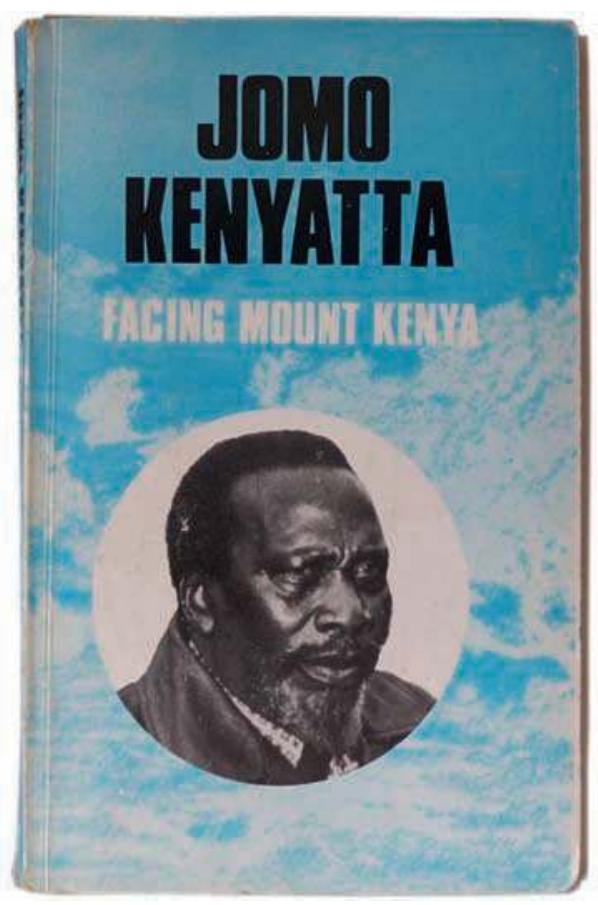

Fig. 5. Première de couverture Facing Mount Kenya, 1978 (Cl. A.-M. Peatrik)

26. Il n'est pas impossible que l'auteur de la version scolaire abrégée, J. Kariuki ait cherché à se faire éditer ailleurs. 
les Embu et les Meru, fiction au fondement d'alliances politiques durables ${ }^{27}$ qui ont nourri tant d'interrogations sur la suprématie des Kikuyu dans l'appareil d'État, est supprimée. Ce qui, en 1938, paraissait aller de soi est devenu sujet à caution, voire dérangeant sans pour autant susciter les explications qu'on serait en droit d'entendre.

Après la première sortie en 1978, les réimpressions à 2000 exemplaires se sont succédé à un rythme soutenu : en 1982, 1984, 1987, 1988, 1989, 1991, 1992, 1994, 1999, 2000, 2002, 2004, 2008, 2011.

Le pic des années 1987-1994 correspond à une redécouverte du Kenya par une nouvelle catégorie d'acheteurs et de lecteurs. Nairobi se transforme en métropole est-africaine, le Kenya à nouveau à la mode attire touristes et expatriés de toutes nationalités, dans le sillage notamment du film Out of Africa sorti en 1985, adaptation par Sydney Pollack de l'ouvrage du même nom écrit par Karen Blixen, dont la maison devenue propriété des National Museums of Kenya a été rénovée et ouverte au public en 1986. Dans Out of Africa, mémoires parues en 1937 (1942 pour l'édition française), Blixen (1885-1962) évoque avec finesse et empathie ses squatters kikuyu. Facing trouve là un nouveau faire-valoir. Ces deux auteurs, des contemporains qui n'ont jamais dû se croiser de leur vivant, entament de la sorte un improbable dialogue post-mortem.

"Facing has done well! » Que ce soit l'édition scolaire ou l'édition intégrale, Facing a été un très bon produit. Parole d'éditeur!

Il faut donc retenir que, à partir des années 1970, le livre, dans une version ou une autre, se diffuse au Kenya dans un contexte où l'habitude de lecture se répand tout autant. Il est lu, simplement parcouru, en tout cas manipulé et feuilleté comme jamais auparavant au Kenya ${ }^{28}$. My People of Kikuyu, réimprimé en 1966 par Oxford Press, accompagne le mouvement. Dans sa courte préface, Kenyatta rappelle le contexte de son écriture en 1942 et avise "Immense tasks of historical study and social recording still remain to be undetaken ». La création de l'Institute of African Studies suivra de peu en 1971, ainsi que le centre culturel Bomas of Kenya, une revanche musicale anticoloniale, chargé de recueillir et de promouvoir les danses africaines en voie de disparition.

27. Il en va ainsi de la célèbre GEMA, acronyme de l'alliance politique dite Gikuyu, Embu, Meru Association, scellée au prétexte que ces peuples seraient des descendants de Moombi, la femme de Gikuyu, une contre-vérité ethnographique promue au rang de fait par nombre de protagonistes, et un très gros dossier ethnologique!

28. En 1966, parait la traduction de Facing en kiswahili chez East African Publishers, association qui cherchait à faire exister une édition africaine, indépendante des puissantes maisons d'édition britanniques (Ogot 2003 : 193-238). Cette édition intitulée Naushangilia Mlima wa Kenya relève de la problématique du statut du kiswahili et de la lecture dans cette langue (recherche en cours). De la même façon, la rumeur d'une traduction en kikuyu de Facing existe, mais je n'en ai toujours pas trouvé d'exemplaire! 


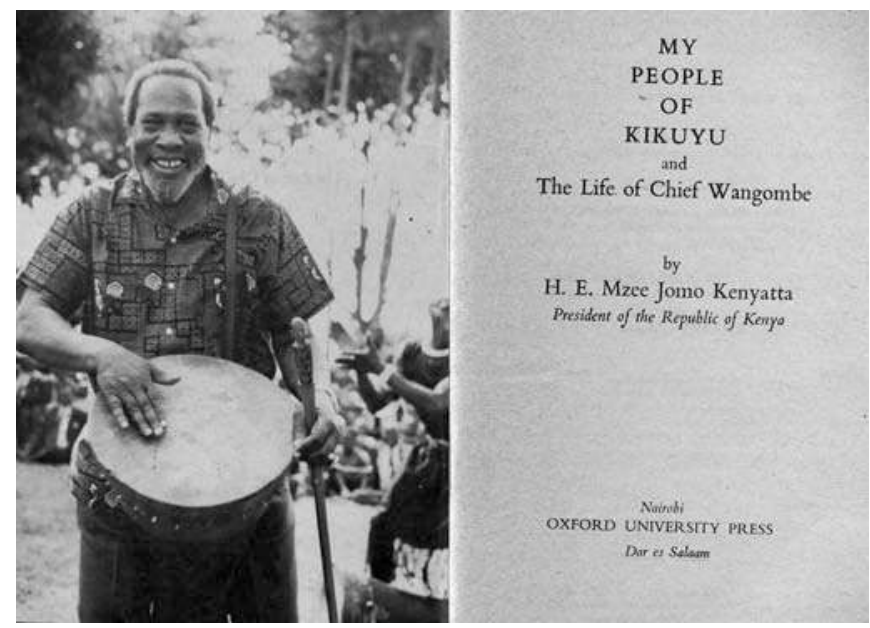

Fig. 6. Frontispice, My People of Kikuyu, 1966 [1942] (Cl. A.-M. Peatrik)

Signe de cette nouvelle célébrité, "Facing quelque chose » devient au Kenya une expression du langage courant, un référent, un marqueur et un démarqueur, mobilisés à des fins diverses dans des titres de livres, les unes de la presse et les slogans ${ }^{29}$, ce qui nous conduit à la dernière étape en date dans la vie de Facing.

\section{Le livre de la discorde nationale}

En raison même de sa diffusion et de sa nouvelle célébrité, Facing pris dans les conflits qui marquent l'entrée du Kenya dans le multipartisme, victime à la longue de lectures simplificatrices propices à la propagation de rumeurs, entame une nouvelle étape dans sa carrière. Comment évoquer en quelques phrases un processus qui émerge en 1988, s'amplifie au rythme des élections générales organisées tous les cinq ans, des changements de président de la république, d'une démographie où la population double à peu près tous les vingt ans? En 1989, les Kenyans étaient 20 millions, en 2009, 40 millions. Daniel arap Moi au pouvoir depuis 1978 (à la mort de Kenyatta), est battu aux élections de 2002 par Mwai Kibaki. La position de ce dernier vascille en 2007, occasionnant les graves affrontements de 2008. En 2013, Kibaki, qui de toute façon ne pouvait plus se représenter, est remplacé par Uhuru Kenyatta, un des jeunes fils du Mzee,

29. Cf. : Obadiah Karuiki, A Bishop Facing Mount Kenya. An Autobiography, 1902-1978 (Nairobi, Uzima Press, 1985) ; Benjamin Kipkorir (Descent From Cherang'any Hills. Memoirs of a Reluctant Academic, Nairobi, Macmillian Kenya, 2009) qui cite le titre par son image inversée; l'article «Is It Yet Uhuru or Should We Be Facing Mt Kenya?» (The Standard, 10 mars 2013), en référence à la confrontation de Raila Odinga et Uhuru Kenyatta, lors des dernières élections présidentielles etc. sont quelques exemples parmi bien d'autres. 
élu de manière quasi régulière. Ces passations, qui tout de même se réalisent - le Kenya occupe une situation géostratégique clé -, se déroulent sur un fond de grande tension où se mêlent débat démocratique porté par une société civile de mieux en mieux organisée et des médias en pleine expansion, affrontements ethniques instrumentalisés par les politiciens en vue des élections, développement économique et inégalités croissantes.

Entre clientélisme et manipulation, des liens de nature fort diverse se sont tissés au fil des ans dans tout le Kenya, entre une hiérarchie politicoadministrative soucieuse de contrôler le pouvoir et de restreindre l'accès au National cake, et des jeunes de plus en plus nombreux, en mal d'intégration, dont certains forment des bandes armées commanditées à l'origine de clash ethniques mortels. Pas moins d'une quarantaine de bandes ont été répertoriées et officiellement interdites.

L'une d'entre elles, apparue dès 1988 dans les districts kikuyu, proches de la capitale Nairobi, est devenue un acteur décisif de la vie politique nationale. Appelés Mûngiki ( les Nombreux») sur le modèle des dénominations de classes générationnelles, organisés initialement en groupe d'autodéfense des Kikuyu pris dans des clashs ethniques et les expulsions foncières, les Nombreux agrègent des effectifs croissants en tirant sur différentes ficelles: se disant héritiers des Mau Mau, ils se présentent comme les défenseurs des pauvres et des redresseurs de tort ${ }^{30}$, et prônent un retour aux traditions telles qu'ils pensent les lire dans Facing, et dont l'oubli serait à l'origine de la crise morale où sombrent tant de jeunes. Synthèse de l'héritage qu'ils revendiquent des Mau Mau et de ce qu'ils pensent être des coutumes des Kikuyu, les Nombreux réclament une passation de pouvoir au profit des plus jeunes, à l'instar de la passation générationnelle (itwika). Au fil des ans, le mouvement devenu nébuleuse se diversifie, la protestation touche à la délinquance, certains cercles entrent dans une culture de la clandestinité et du racket, les pratiques de violence s'accentuent, notamment l'élimination physique des opposants, et les Mûngiki finissent par être infiltrés par des services spéciaux. Les émeutes de 2007-2008 qui suivent les résultats très contestés des élections de 2007, se traduisent par un millier de morts et 500000 réfugiés de l'intérieur. Au pré-procès instruit par le Tribunal pénal international de La Haye saisi pour crime contre l'humanité, apparaissent le nom des Mûngiki, conjointement à celui de Uhuru Kenyatta et de William Ruto, tous deux vainqueurs des élections présidentielles de 2013.

30. À l'appui de cette relecture, il faut aussi évoquer les travaux des nouveaux historiens qui ont étudié la répression menée par l'armée britannique et l'administration coloniale contre les Mau Mau. Ils confortent l'idée que les Mau Mau, sans lesquels l'indépendance n'aurait pu être obtenue, ont été trahis et demeurent sans terre et doivent exiger des réparations (Charton 2011). 
Facing est donc pris dans cette nouvelle tempête ! Brandi symboliquement par les Mûngiki comme garant de l'identité des Kikuyu, Facing mué en symbole de l'égoïsme ethnique et de l'hégémonie d'une seule tribu, devient le livre de la discorde nationale. On raconte que Uhuru Kenyatta aurait activement fait circuler et distribuer des exemplaires. Il est vrai qu'on trouve maintenant dans les librairies l'édition de Facing, produite aux États-Unis par Vintage avec la photographie de Javelot flamboyant en couverture et la fameuse carte du pays kikuyu. Éditions Vintage la bien nommée, entre rétromanie et nostalgie: le décalage temporel introduit par l'édition de Heinemann/Chakava qui indiquait une profondeur historique, une mise à distance, est aboli.

Les études sur les Mûngiki devenus une nébuleuse sont en cours de réalisation ${ }^{31}$. Le phénomène est complexe à analyser, dangereux aussi, on touche aux affaires où les services spéciaux sont impliqués, des chercheurs ont été inquiétés. On peut cependant s'interroger sur la nouvelle

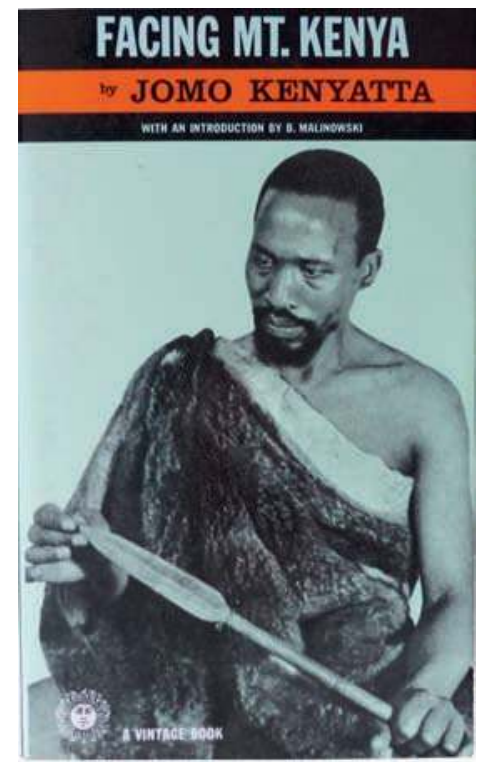

Fig 7. Première de couverture, Facing Mount Kenya, 1965 (“Vintage Book") (Cl. A.-M. Peatrik) lecture de Facing qu'auraient imposée les Mûngiki. Que la détention d'un exemplaire de Facing soit valorisée, c'est vraisemblablement le cas. Quid cependant de la lecture effective de Facing par la masse des Mûngiki ? Le livre est long, touffu, il suppose une concentration du lecteur et un certain savoir-faire analytique. La dégaine de nombre d'activistes cadre mal avec la posture du lecteur durablement concentré.

Cet article est une contribution à l'histoire de l'ethnologie par le biais de la biographie d'une monographie, de la matérialité et de la réception d'un ouvrage dont l'auteur a fini par devenir un homme politique de stature internationale. Quelle eût été la carrière de Kenyatta en l'absence de Facing? Quelle eût été la renommée de Facing en l'absence de la carrière politique de son auteur? Le contenu informatif du texte en aurait probablement été mieux reconnu mais le texte n'aurait jamais atteint le statut international qu’il a gagné dans les années 1960, illustratif aussi de la

31. Cf. : David Anderson (2002); Peter Kagwanja (2003); Hervé Maupeu (2002); Jérôme Lafargue (2010). 
notoriété acquise alors par l'ethnologie. Que Jomo se soit servi de son image d'auteur pour construire sa carrière politique, nul n'en doute, il ne serait pas le premier. Faut-il pour autant pousser le déterminisme en réduisant le contenu de l'ouvrage à ce carriérisme et aux lectures postérieures qui en seront faites? Nous voilà donc devant un bien étrange objet, dont il fallait reconstituer l'histoire de vie, au cours de laquelle se sont détachés des doubles et fantômes, textes en miroir qui poussent à une exploration supplémentaire. Au nom d'une histoire de la littérature ou de la pensée ? Pas exactement : l'enjeu est encore celui de la politique des connaissances, ethnologiques, et de la maîtrise de leur passé et de leurs sources ethnographiques par les peuples concernés. Plus largement, cette recherche vise à définir des nouveaux objets et à envisager des questionnements inédits dans le domaine très prometteur, et en voie de constitution, de l'anthropologie des savoirs.

Centre national de la recherche scientifique, Université Paris Ouest Nanterre La Défense Laboratoire d'ethnologie et de sociologie comparative, Nanterre anne-marie.peatrik@mae.u-paris10.fr

MOTS CLÉS/KEYWORDS: histoire de l'ethnologie/history of ethnology - Afrique de l'Est/East Africa - Kenya - Jomo Kenyatta - Facing Mount Kenya - Bronislaw Malinowski - Kikuyu Mau Mau - âges et génération/ages and generation - édition/publication - lectorat/readership - anthropologie des savoirs/anthropology of knwoledge. 
Tableau comparatif des éditions de Facing, I938 et |97|

Jomo Kenyatta 1938

Facing Mount Kenya. The Tribal Life of the Gikuyu (339 p.)

Introduction by B. Malinowski VII-XIV

Preface by Jomo Kenyatta XIV-XXI

Ch I Tribal Origin and Kinship System 1-19

Ch 2 System of Land Tenure 20-52

Ch 3 Economic Life of the Gikuyu 53-59

Ch 4 Industries 70-97

Ch 5 Systems of Education (Prior to the Advent of the European) 98-129

Ch 6 Initiation of Boys and Girls 130-154

Ch 7 Sex Life Among Young People 155-162

Ch 8 Marriage System 163-185

Ch 9 The Gikuyu System of Government 186-230

Ch 10 Gikuyu Religion, Ancestor Worship and Sacrificial Practices 23I-268

Ch 1 I The New Religion in East Africa 269-279

Ch 12 Magical \& Medical Pratices 280-308

Ch 13 Conclusion 309-318

Glossary 319-329

Index 331-339

School Edition 1971

Facing Mount Kenya.The Tribal Life of the Gikuyu (169 p.)

Foreword by His Excellency Mzee Jomo Kenyatta,

President of the Republic of Kenya

Ch I Tribal Origin and Kinship System I-I।

Ch 2 Gikuyu System of Land Ownership |3-3|

Ch 3 Agriculture $32-40$

Ch 4 Industries 4I-59

Ch 5 Systems of Education 60-74

Ch 6 Initiation of Boys and Girls 75-86

Ch 7 Marriage System 87-98

Ch 8 Gikuyu System of Government 99-125

Ch 9 Gikuyu Religion 127-148

Ch 10 Magical \& medical Pratices 149-169

Ch II Conclusion 166-169 
Anderson, David

2002 "Vigilantes, Violence and the Politics of Public Order in Kenya ", African Affairs $101: 531-555$.

Asad, Talal, ed.

1973 Anthropology and the Colonial

Encounter. London, Ithaca Press/

Atlantic Higlands, Humanities Press.

Balandier, Georges, Georges Steinmetz

\& Gisèle Sapiro

2011 "Tout parcours scientifique comporte des moments autobiographiques", Actes de la recherche en sciences sociales 185 (5) : 44-61.

Barlow, Arthur R.

1939 "Review of Facing Mount Kenya", Africa 12 (1) : 114-116.

\section{Beck, Ann}

1966 "Some Observations on Jomo

Kenyatta in Britain 1929-1930 ",

Cahiers d'études africaines 6 (22) : 308-329

[http://www.persee.fr/web/revues/

home/prescript/article/

cea_0008-0055_1966_num_6_22_3068].

\section{Berman, Bruce}

1996 «Ethnography as Politics, Politics as Ethnography: Kenyatta, Malinowski, and the Making of Facing Mount Kenya", Canadian Journal of African Studies 30 (3) : 313-344.

\section{Berman, Bruce J. \& John M. Lonsdale}

1991 «Louis Leakey's Mau Mau :

A Study in the Politics of Knowledge ", History and Anthropology 5 (2) : 143-204.

1998 «The Labors of Mwigwithania : Jomo Kenyatta as Author 1928-1945 ", Research in African Literatures 1 : 16-42.

2007 "Custom, Modernity, and the Search for Kihooto: Kenyatta, Malinowski and the Making of Facing Mount Kenya", in Helen Tilley \& Robert J. Gordons, eds, Ordering
Africa, Anthropology, European Imperialism and the Politics of Knowledge. ManchesterNew York, Manchester University Press : 173-198.

Blixen, Karen

1942 La Ferme africaine. Traduit du danois par Yvonne Manceron. Paris, Gallimard [titre orig. : Out of Africa, 1937].

Boukari-Yabara, Amzat

2014 «Les militants noirs anglophones des années 1920 à 1940 », Gradhiva 19 : 30-51.

Buijtenhuijs, Robert

1971 Le Mouvement "Mau Mau». Une révolte paysanne et anti-coloniale en Afrique noire. La Haye-Paris, Mouton.

Celarent, Barbara

(pseud. de Andrew Delano Abbott)

2010 «Facing Mount Kenya by Jomo Kenyatta ", American Journal of Sociology 116 (2) : 722-728

[http://home.uchicago.edu/aabbott/ barbpapers/barbkenya.pdf].

\section{Charton, Hélène}

2011 «Acteurs, victimes et témoins de la violence dans l'histoire : l'exemple mau mau (Kenya)", Cahiers d'études africaines 201 (1) : 169-182.

Cohen, Anouk

2011 Fabriquer le livre à Rabat et à Casablanca. Une ethnographie. Nanterre, Université Paris Ouest Nanterre La Défense, thèse de doctorat.

Copans, Jean, ed.

1975 Anthropologie et impérialisme.

Paris, François Maspéro.

Corfield, Franck

1960 The Origins and Growth of Mau Mau. An Historical Survey (= "The Corfield Report»). Nairobi, Government of Kenya. 
Doke, C. M.

1938 "Review of Facing Mount Kenya", Bantu Studies 12 : 245-246.

\section{Fortes, Meyer \&}

Edward Evans-Pritchard, eds

1940 African Political Systems.

London, Oxford University Press

for the International African Institute.

\section{Frederiksen, Bodil Folke}

2008 "Jomo Kenyatta, Marie Bonaparte and Bronislaw Malinovski

on Clitoridectomy and Female Sexuality ", History Workshop Journal 65 (1) : 23-48.

\section{Goody, Jack}

1995 The Expansive Moment. The Rise of Social Anthropology in Britain and Africa. Cambridge, Cambridge University Press.

James, Wendy

1973 "The Anthropologist as Reluctant Imperialist ", in Talal Asad, ed., Anthropology and the Colonial Encounter... : 41-69.

Jamin, Jean

1973 Contacts et antagonismes culturels en pays kikuyu (Kenya). Paris, Institut d'ethnologie.

1977 Les Lois du silence. Essai sur la fonction sociale du secret. Paris, François Maspero («Dossiers africains»).

\section{Kagwanja, Peter Mwangi}

2003 «Facing Mount Kenya or Facing Mecca? The Mungiki, Ethnic Violence and the Politics of the Moi Succession in Kenya, 1987-2002 ", African Affairs 102: 25-49.

\section{Kenyatta, Jomo}

1937 «Kikuyu Religion, Ancestor-

Worship, and Sacrificial Practices ", Africa 10 (3) : 308-328.

1938 Facing Mount Kenya.

The Tribal Life of the Kikuyu.

With an introd. by Bronislaw Malinowski. London, Secker \& Warburg.
1942 My People of Kikuyu and the Life of Chief Wangombe. London, United Society for Christian Literature.

1945 Kenya. The Land of Conflict.

London, International African Service Bureau Publication.

1960 Au pied du Mont Kenya.

Préf. de Georges Balandier. Paris, Maspero ("Les Textes à l'appui»).

1965 Facing Mount Kenya. The Tribal Life of the Kikuyu. New York, Random House ("Vintage Book»).

1966a My People of Kikuyu and the Life of Chief Wangombe, by H. E. Mze Jomo Kenyatta. Nairobi-London, Oxford University Press.

1966b Naushangilia Mlima wa Kenya.

Nairobi, East African Publishing House.

1967 Au pied du Mont Kenya.

Préf. augmentée de Georges Balandier.

Paris, Éditions Maspero ( Petite collection François Maspero").

1968 Suffering Without Bitterness.

The Founding of the Kenyan Nation.

Nairobi, East African Publishing House.

1971 Facing Mount Kenya. The Tribal Life of the Kikuyu. Ed. by J. Kariuki. Nairobi, Heinemann Educational Books.

1973 Au pied du mont Kenya. Paris, Le Club Africain du Livre-François Maspero.

1976 La Montagna dello Splendore.

Per un'antropologia dell'Africa nera. Milano, Jaca Book [rééd. en 1990 par Arnoldo Mondadori Editore].

1978 Facing Mount Kenya.

The Tradional Life of the Kikuyu.

Nairobi, Heinemann Kenya.

\section{Kershaw, Greta}

1972 The Land is the People. A Study of Kikuyu Social Organisation in Historical Perspectives. Chicago, University of Chicago, unpublished $\mathrm{PhD}$.

1997 Mau Mau from Below. Oxford, James Currey/Nairobi, EAEP/Athens, Ohio University Press (« Eastern African Studies »). 


\section{Kopytoff, lgor}

1985 "The Cultural Biography of Things :

Commoditization as Process ", in Arjun

Appadurai, ed., The Social Life of Things.

Commodities in Cultural Perspective.

Cambridge, Cambridge University Press : 64-91.

Kuper, Adam

2000 [1996] L'Anthropologie britannique au XXe siècle. Trad. de l'anglais par Gérald Gaillard. Paris, Karthala ( Hommes et sociétés") [titre orig. : Anthropology and Anthropologists. The Modern Bristish School].

Lafargue, Jérôme

2010 "Résistances au long cours : narration et mémoire insurrectionnelle à partir de l'exemple des Mau Mau (Kenya)", Cahiers d'études africaines 197 (1) : 25-50.

Lambert, Harold Ernest

1956 Kikuyu Social and Political

Institutions. London-New York, Oxford

University Press for the International

African Institute.

Lanternari, Vittorio

1962 Les Mouvements religieux de liberté et de salut des peuples opprimés.

Trad. de l'italien par Robert Paris. Paris, François Maspero («Les Textes à l'appui»).

Leakey, Louis Seymour Bazett

1931 "The Kikuyu Problem of the Initiation of Girl ", The Journal of the Royal Anthropological Institute of Great Britain and Ireland $61: 277-285$.

1952 Mau Mau and the Kikuyu.

London, Methuen.

1954 Defeating Mau Mau.

London, Methuen.

1977 The Southern Kikuyu Before 1903.

London-New York, Academic Press. 3 vol.

Leclerc, Gérard

1972 Anthropologie et colonialisme.

Essai sur l'histoire de l'africanisme.

Paris, Fayard («Anthropologie critique»).
L'Estoile, Benoît de

1997 "Au nom des "vrais Africains": les élites scolarisées de l'Afrique coloniale face à l'anthropologie (1930-1950)", Terrain $28: 87-102$

[http://terrain.revues.org/3173].

Lindfors, Bernth

2011 Early East African Writers and Publishers. Trenton-Asmara, Africa World Press.

McClellan, Woodford

1993 "Africans and Black Americans in the Comintern Schools, 1925-1934 ", International Journal of African Historical Studies 26 (2) : 371-390.

Malinowski, Bronislaw

1929 "Practical Anthropology", Africa 2 (1) : 22-38.

Maspéro, François

2002 Les Abeilles et la guêpe.

Paris, Le Seuil («Point»).

Maupeu, Hervé

2002 "Mungiki et les élections :

les mutations politiques d'un prophétisme kikuyu ", Politique africaine 87 : 117-137 [http://www.politique-africaine.com/ numeros/pdf/087117.pdf].

Mauss, Marcel

1971 [1931] «La cohésion sociale dans les sociétés polysegmentaires", in Essais de sociologie. Paris, Minuit : 133-147.

Middleton, John

1953 The Kikuyu and Kamba of Kenya. London, International African Institute ("Ethnographic Survey of Africa»). 1954 Les Kikouyous et les Kambas du Kénia. Étude scientifique sur les Mau-Mau. Paris, Payot.

Middleton, John \& Greet Kershaw 1965 The Central Tribes of the NorthEastern Bantu (The Kikuyu, including Embu, 
Meru, Mbere, Chuka, Mwimbi, Tharaka, and the Kamba of Kenya). London,

International African Institute

(«Ethnographic Survey of Africa »).

Middleton, John \& David Tait

1958 Tribes Without Rulers.

Studies in African Segmentary Systems.

London, Routledge \& Kegan Paul.

Mills, David

2008 Difficult Folk? A Political History of Social Anthropology. New York, Berghahn Books (" Methodology and History in Anthropology »).

Mockerie, Parmenas Githendu

1934 An African Speaks for his People. London, Leonard \& Virginia Woolf at the Hogarth Press.

Mühlman, Wilhelm E.

1968 [1961] Messianismes révolutionnaires $d u$ tiers-monde. Trad. de l'allemand par Jean Baudrillard. Paris, Gallimard ("Bibliothèque des sciences humaines").

Muriuki, Godfrey

1974 A History of the Kikuyu, 1500-1900. Nairobi, Oxford University Press.

Murray-Brown, Jeremy

1972 Kenyatta. London, Allen \& Unwin.

Nizan, Paul

1969 [1932] Les Chiens de garde.

Paris, François Maspero

(«Petite collection Maspero »).

Ntarangwi, Mwenda, David Mills

\& Mustapha Babiker, eds

2006 African Anthropologies. History,

Critique and Practice. London-New York, Zed Books/Dakar, Codesria.

Ogot, Bethwell Allan

2003 My Footprints on the Sands of Time. An Autobiography. Victoria, Trafford Publishing.
Ozouf, Jacques \& Mona Ozouf

1997 [1984] «Le Tour de la France par deux enfants : le petit livre rouge

Les Lieux de mémoire, 1. Paris, Gallimard ("Quarto») : 277-302.

Peatrik, Anne-Marie

1994 «Un système composite :

l'organisation d'âge et de génération des Kikuyu pré-coloniaux ", Journal des africanistes 64 (1) : 3-36

[http://www.persee.fr/web/revues/ home/prescript/article/jafr_ 0399-0346_1994_num_64_1_2390].

1995 «La règle et le nombre: les systèmes d'âge et de génération en Afrique orientale ", L'Homme 134 (2) : 13-49 [http://www.persee.fr/web/revues/ home/prescript/article/hom 0439-4216_1995_num_35_134_369906].

1999 La Vie à pas contés. Génération, âge et société dans les hautes terres du Kénya (Meru Tigania-Igembe, Kénya). Nanterre, Société d'ethnologie ("Sociétés africaines »).

\section{Peterson, Derek R.}

2004 Creative Writing. Translation, Bookkeeping and the Work of Imagination in Colonial Kenya. Portsmouth, Heinemann («Social History of Africa»).

Prins, Adriaan $\mathrm{H}$.

1953 East African Age-Class Sytems. An Inquiry into the Social Order of Galla, Kipsigis and Kikuyu. Groningen,

J. B. Wolters.

Ribero, Gustavo Lins

\& Arturo Escobar, eds

2006 World Anthropologies. Disciplinary Transformations Within Systems of Power. Oxford-New York, Berg.

Ricard, Alain

1995 Littératures d'Afrique noire.

Des langues aux livres.

Paris, Éd. du CNRS-Karthala. 


\section{Rossetti, Carlo Giuseppe}

1985 «B. Malinowski, the Sociology of "Modern Problems" in Africa and the "Colonial Situation" ", Cahiers d'études africaines 100 (4) : 477-503

[http://www.persee.fr/web/revues/home/ prescript/article/cea_0008-

0055_1985_num_25_100_1719].

Soga, John Henderson

1930 The South-Eastern Bantu

(Abe-Naguni, Aba-Mbo, Ama-Lala).

Johannesburg, Witwatersrand

University Press.

Tilley, Helen

2011 Africa as a Living Laboratory.

Empire, Development, and the Problem

of Scientific Knowledge 1870-1950.

Chicago, University of Chicago Press.
Tilley, Helen

\& Robert J. Gordon, eds

2007 Ordering Africa. Anthropology,

European Imperialism and the Politics

of Knowledge. Manchester-New York,

Manchester University Press.

Young, Cullen

1938 "Review of Facing Mount Kenya", Journal of the Royal African Society 37

(149) : 522-523.

Warburg, Frederic

1959 An Occupation for Gentlemen.

London, Hutchinson

1973 All Authors are Equal.

The Publishing Life of Frederic Warburg,

1936-1971. London, Hutchinson.
Anne-Marie Peatrik, Le singulier destin de Facing Mount Kenya.The Tribal Life of Gikuyu (1938) de Jomo Kenyatta: une contribution à l'anthropologie des savoirs. — L'article est une contribution à l'histoire de l'ethnologie et à l'anthropologie des savoirs par le truchement de la biographie de la première monographie d'ethnologie réalisée at home par un sujet colonial, Jomo Kenyatta qui devint en 1964 le premier président du Kenya indépendant. Après avoir rappelé l'origine de ce texte - un master d'anthropologie soutenu à la London School of Economics sous la direction de Bronislaw Malinowki et publié en 1938 - on abordera la question de sa réception paradoxale, au travers des éditions successives dont l'ouvrage a fait l'objet. Tantôt considéré comme la vision fonctionnaliste d'une société idéalisée, tantôt décrié ou célébré comme manifeste anticolonial, tantôt encore brocardé comme texte de la division nationale, ce texte a rarement été lu pour ce qu'il est d'abord, une source ethnographique pour la connaissance des Kikuyu et des peuples d'Afrique de l'Est.
Anne-Marie Peatrik, The Singular Destiny of Facing Mount Kenya. The Tribal Life of the Gikuyu (1938) by Jomo Kenyatta: A Contribution to the Anthropology of Knowledge. - The article is a contribution to the history of ethnology and the anthropology of knowledge with an in-depth review of the first ethnological monograph completed " at home " by a colonial subject, Jomo Kenyatta, who in 1964 became the first president of an independent Kenya. After a look at the origin of the book - a master's thesis in anthropology defended at the London School of Economics under the supervision of Bronislaw Malinowki, and published in 1938 - the article examines the issue of its paradoxical reception with each successive publication of the work. At times considered the functionalist vision of an idealised society, at others decried or celebrated as an anti-colonial manifesto, at others yet derided as a textbook of national division, the work has hardly ever been read for what it originally meant to be - an ethnographic source for the understanding of the Kikuyu and East African people. 